Research Article

\title{
Evaluation Method of Wind Speed Time-Shifting Characteristics at Multiple Scales and Its Application in Wind Power System
}

\author{
Han Wang $\mathbb{D}^{\mathbb{D}}$, Shuang Han $\mathbb{D}$, Yongqian Liu, and Aimei Lin \\ State Key Laboratory of Alternate Electrical Power System with Renewable Energy Sources, School of Renewable Energy, \\ North China Electric Power University (NCEPU), Beijing 102206, China \\ Correspondence should be addressed to Shuang Han; hanshuang1008@sina.com
}

Received 31 July 2020; Accepted 23 October 2020; Published 21 November 2020

Academic Editor: Haoran Zhang

Copyright (c) 2020 Han Wang et al. This is an open access article distributed under the Creative Commons Attribution License, which permits unrestricted use, distribution, and reproduction in any medium, provided the original work is properly cited.

The wind speed sequences at different spatial positions have a certain spatiotemporal coupling relationship. It is of great significance to analyze the clustering effect of the wind farm(s) and reduce the adverse impact of large-scale wind power integration if we can grasp this relationship at multiple scales. At present, the physical method cannot optimize the timeshifting characteristics in real time, and the research scope is concentrated on the wind farm. The statistical method cannot quantitatively describe the temporal relationship and the speed variation among wind speed sequences at different spatial positions. To solve the above problems, a quantification method of wind speed time-shifting characteristics based on wind process is proposed in this paper. Two evaluation indexes, the delay time and the decay speed, are presented to quantify the time-shifting characteristics. The effectiveness of the proposed method is verified from the perspective of the correlation between wind speed sequences. The time-shifting characteristics of wind speed sequences under the wind farms scale and the wind turbines scale are studied, respectively. The results show that the proposed evaluation method can effectively achieve the quantitative analysis of time-shifting and could improve the results continuously according to the actual wind conditions. Besides, it is suitable for any spatial scale. The calculation results can be directly applied to the wind power system to help obtain the more accurate output of the wind farm.

\section{Introduction}

A high proportion of renewable energy is one of the basic characteristics of the future electric power system, and wind power is an important component of renewable energy [1]. With the rapid expansion of wind power integration, the volatility of wind power has a more adverse impact on the grid dispatching, safe and stable operation of the electric power system, and its power quality [2]. In fact, there is a certain spatiotemporal coupling relationship among the wind speed sequences at different spatial points [3]. If we can grasp this coupling relationship at multiple temporal-spatial scales, it is of great significance to analyze the clustering effect [4], deal with the ramp events and extreme events, obtain the real output of wind farm(s) [5], and so forth, which can effectively reduce the negative effect of the wind power integration [6].
At present, the research algorithms on the spatiotemporal coupling relationship among wind speed time series can be divided into two categories, the model-based physical method and the data-based statistical method, according to the driving mode.

The physical method takes the wind speed data at a certain point as input and considers factors such as terrain, roughness, altitude, and flow to establish the aerodynamic model to describe the coupling relationship among the wind speeds at different spatial locations. Zheng [7] assumed that the wind spreads at the speed of the starting point and established the motion equation of wind speed to obtain the average delay time between the wind speed time series at two spatial positions. She et al. [8] used the rotor diameter and geographic coordinate matrix of each wind turbine in the wind farm as the inherent characteristics and took the wind 
speed and direction of the wind tower, rotor speed, and pitch angle of each wind turbine as inputs to establish the wake effect model and the time-lag effect model; then the dynamic spatial relationship among wind speed time series at each wind turbine location was obtained. Zeng et al. [9] first developed the continuous partial differential equation of each wind turbine by calculating the wake effects, according to the specific spatial locations and distributions of correlated wind turbines. Then, the finite volume method is used to differentiate the differential equation at each grid point and derive the spatial correlation matrix of wind speed. Finally, the wind speed at each wind turbine location is obtained by solving the above differential equation through the given boundary conditions. Ye et al. [10] proposed a novel spatial correlation matrix by using computational fluid dynamics to mathematically describe the correlations among wind speeds at different wind turbine locations.

The physical method studies the temporal and spatial distribution and the coupling relationship of wind speeds by establishing the aerodynamic model among the wind speeds at different spatial positions. However, this aerodynamic model cannot be optimized in real time according to the actual wind conditions. Besides, when the spatial scale increases, the complexity of the constructed model increases sharply, which leads to the increasing difficulty of modeling and solving the model simultaneously. But it is difficult to ensure the accuracy of the aerodynamic model if the factors affecting wind speed are simplified. Therefore, the application of this method is mainly concentrated in the interior of the wind farm, which is difficult to expand to a larger spatial scale.

The statistical method uses the actual wind data at different spatial locations to analyze the spatiotemporal coupling relationship among the wind speeds. The research methods can be mainly divided into the following three types:

(1) The spatiotemporal coupling relationship is studied by calculating the distribution of wind speeds at different spatial locations, from the perspectives of average wind speed, wind speed frequency distribution, wind shear exponent, and so forth [11]. Liu et al. [12] analyzed the spatiotemporal distributions of the wind resource at the hub height in China from the aspects of average wind speed and average wind power density, based on the large network of wind speed observations, including 2430 meteorological stations from 2006 to 2015. The results show that northeast China has the highest wind speed, while South China has the lowest wind speed. $\mathrm{Li}$ and $\mathrm{Yu}$ [13] conducted the statistical analysis of the onshore, nearshore, and offshore wind energy conditions from the aspects of Weibull shape and scale factors, average turbulence intensity, and average wind power density. The results indicate that the average wind speed at the offshore location is 1-2 times higher than that nearby onshore area at the height of $50 \mathrm{~m}$ above ground level. Bosch et al. [14] estimated the distributions of global offshore wind energy potential from the aspects of average wind speed, mean capacity factor which could be built across the viable offshore area, and generation potential per year. The results show that the wind speed increases with the distance to the coast.

(2) The spatiotemporal coupling relationship is studied by establishing the mapping relationship among wind speed time series at different spatial positions through interpolation, neural network, principal component analysis, and so forth [15]. Philippopoulos and Deligiorgi [16] used two feedforward neural network architectures to establish the relationship among hourly wind speeds at six meteorological sites. An insight into the underlying inputoutput function approximation of the neural networks is obtained by examining their ability to incorporate the mean wind variability characteristics. Miranda and Dunn [17] proposed a spatially correlated wind speed model to characterize the wind resources between various sites by using the multivariate time-series approach with actual wind speed data in different zones. Park and Jang [18] presented a modified Korean parameter-elevation regression on independent slopes model for spatial interpolation of monthly wind speeds. The results show that the proposed model can perform more appropriately to represent the phenomena where similar wind speeds appear continuously along ridges and coastlines, compared with the cokriging model.

(3) The correlation coefficient is used to study the correlation characteristics of wind speed time series at different spatial positions, which is an important part of the spatiotemporal coupling relationship. The calculation method can be divided into the following categories. One is to directly calculate the correlation coefficient based on the wind speed time series [19]. Another is to use the Copula function to fit the joint probability distribution of the wind speed sequences and then calculate the correlation coefficient based on the fitted Copula function [20]. The commonly used correlation coefficients include the Pearson linear correlation coefficient [21], Kendall rank correlation coefficient [22], and cross-correlation coefficient [23].

The statistical method studies the spatiotemporal coupling relationship of wind speed sequences at different spatial positions based on the actual wind data. The obtained results of this method can be continuously improved according to actual conditions, and the research method can be applied to any spatial scale. However, only the correlation relationship between wind speed sequences is quantified in this method. Compared with the physical method, the temporal relationship and the law of speed variation are both implicit in the spatial distribution, and the mapping relationship is not quantified yet.

In conclusion, when the physical method is used to study the spatiotemporal coupling relationship among wind speeds at different spatial positions, the established aerodynamic model cannot be optimized in real time according 
to the actual wind conditions, and the application of this method mainly concentrated in the interior of the wind farm; it is difficult to expand to a larger spatial scale. When the statistical method is applied, only the correlation relationship of wind speed sequences is quantified, and the temporal relationship and the law of speed variation among wind speed sequences at different spatial positions have not been quantified yet. In order to solve the above problems, a quantification method of time-shifting characteristics based on wind process is proposed in this paper. The results of the proposed method can be continuously improved according to the actual wind conditions and could be applied to any spatial scale. The main contributions of this paper are as follows:

(1) The concept of wind process is introduced to study the time-shifting characteristics. In order to cope with the complexity and variability of wind conditions, the wind sequences at different spatial positions are divided according to the certain time window at first, and then the time-shifting characteristics are studied based on the wind processes.

(2) Two evaluation indexes, the delay time and the decay speed, are proposed to quantify the time-shifting characteristics of the temporal relationship and the speed variation in each wind process.

(3) The time-shifting characteristics are studied at two spatial scales, the wind farms scale and the wind turbines scale.

(4) The improved clustering method of $k$-means, $x$ means, is employed to extract the typical wind processes to explore the time-shifting characteristics among wind speeds under various typical wind processes. The obtained results can be directly used in the wind power system.

(5) The validity and practicability of the proposed method are verified by applying the calculation results of time-shifting among wind speed sequences at different spatial positions to the wind power system.

The remainder of this paper is organized as follows. Section 2 describes the research process of wind speed timeshifting. Section 3 studies the wind speed time-shifting characteristics under two spatial scales, the wind farms scale and the wind turbines scale. Section 4 verifies the effectiveness and practicability of the proposed time-shifting evaluation method. Section 5 concludes this paper.

\section{Research Process of Wind Speed Time- Shifting}

This section proposes an evaluation method of the timeshifting characteristics among wind speed sequences at different spatial positions. The research process is as follows.

2.1. Outline of the Research Process on Wind Speed TimeShifting. First, the wind sequence at each point is preprocessed. The wind sequence is divided according to a certain time window to obtain the wind processes at each point. Then some wind processes are screened out to compose a new wind sequence that can be used to study the time-shifting characteristics by judging whether the main wind direction in the wind process is consistent with the relative position of points. For example, the wind sequence at point $p$ can be expressed as a composed sequence of wind process $\alpha_{p}$, wind process $\beta_{p}$, wind process $m_{p}$, and so forth.

Then, two evaluation indexes, the delay time $(\Delta t)$ and the decay speed $(\Delta v)$, are proposed to study the time-shifting characteristics between wind processes at two points. The firefly optimization algorithm is used to calculate the proposed indexes, in which the constraint conditions are the distance between two points and the actual wind conditions; the optimal objective is the minimum Euclidean distance between the corresponding wind processes. Taking the wind process $\alpha_{p}$ and the wind process $\alpha_{q}$ as an example, the wind process $\alpha_{p}$ at point $p$ and the wind process $\alpha_{q}$ at point $q$ can be expressed as $v_{p}^{\alpha}(t-x), \ldots, v_{p}^{\alpha}(t), \ldots, v_{p}^{\alpha}(t+y)$ and $v_{q}^{\alpha}(t-x), \ldots, v_{q}^{\alpha}(t), \ldots, v_{q}^{\alpha}(t+y)$, respectively. The distance between two points and the wind conditions of the wind process $\alpha_{p}$ at point $p$ are set as the constraint conditions, and the wind process $\alpha_{q}$ is offset through the time axis and the speed axis to achieve the objective of the minimum Euclidean distance between the wind process $\alpha_{p}$ at point $p$ and the new wind process $\alpha_{q}^{\prime}$ at point $q$. Then the corresponding delay time $\Delta t_{\alpha}$, decay speed $\Delta v_{\alpha}$, and the new wind process $\alpha_{q}^{\prime}\left(v_{q}^{\alpha}(t-\right.$ $\left.x+\Delta t_{\alpha}\right)+\Delta v_{\alpha}, \ldots, v_{q}^{\alpha}\left(t+\Delta t_{\alpha}\right)+\Delta v_{\alpha}, \ldots, v_{q}^{\alpha}\left(t+y+\Delta t_{\alpha}\right)+$ $\Delta v_{\alpha}$ ) could be obtained at the same time.

Finally, the time-shifting characteristics of wind speed sequences at two points are studied based on the proposed indexes. The delay time and the decay speed of each wind process at point $p$ and point $q$ are calculated, and then the statistical analysis method is used to analyze the timeshifting. Furthermore, the improved clustering method of $k$ means, $x$-means, is employed to extract the typical wind processes, and the time-shifting characteristics of different typical wind processes are studied so that the research results can be applied to the actual planning and operation of the electric power system.

In order to verify the effectiveness of the proposed timeshifting analysis method, the wind speed sequence at point $q$ is reconstructed after considering the time-shifting characteristics. t-Copula function is used to fit the joint probability distribution between wind speed sequences under two conditions, that is, whether the time-shifting is considered. Then the Kendall rank correlation coefficient $\tau$, the Spearman rank correlation coefficient $\rho_{s}$, and the tail correlation coefficient $\lambda$ are calculated based on the t-Copula function. The correlation coefficients increase with the increasing similarity between two wind speed sequences.

The outline of the research process on wind speed timeshifting is shown in Figure 1.

It should be noted that, due to the fact that the wind speed can gradually recover within a certain distance after passing through the wind turbine(s) or the wind farm(s), the evaluation indexes for time-shifting analysis should be different according to the distance and the installed capacity of wind power, that is, whether the decay speed should be 


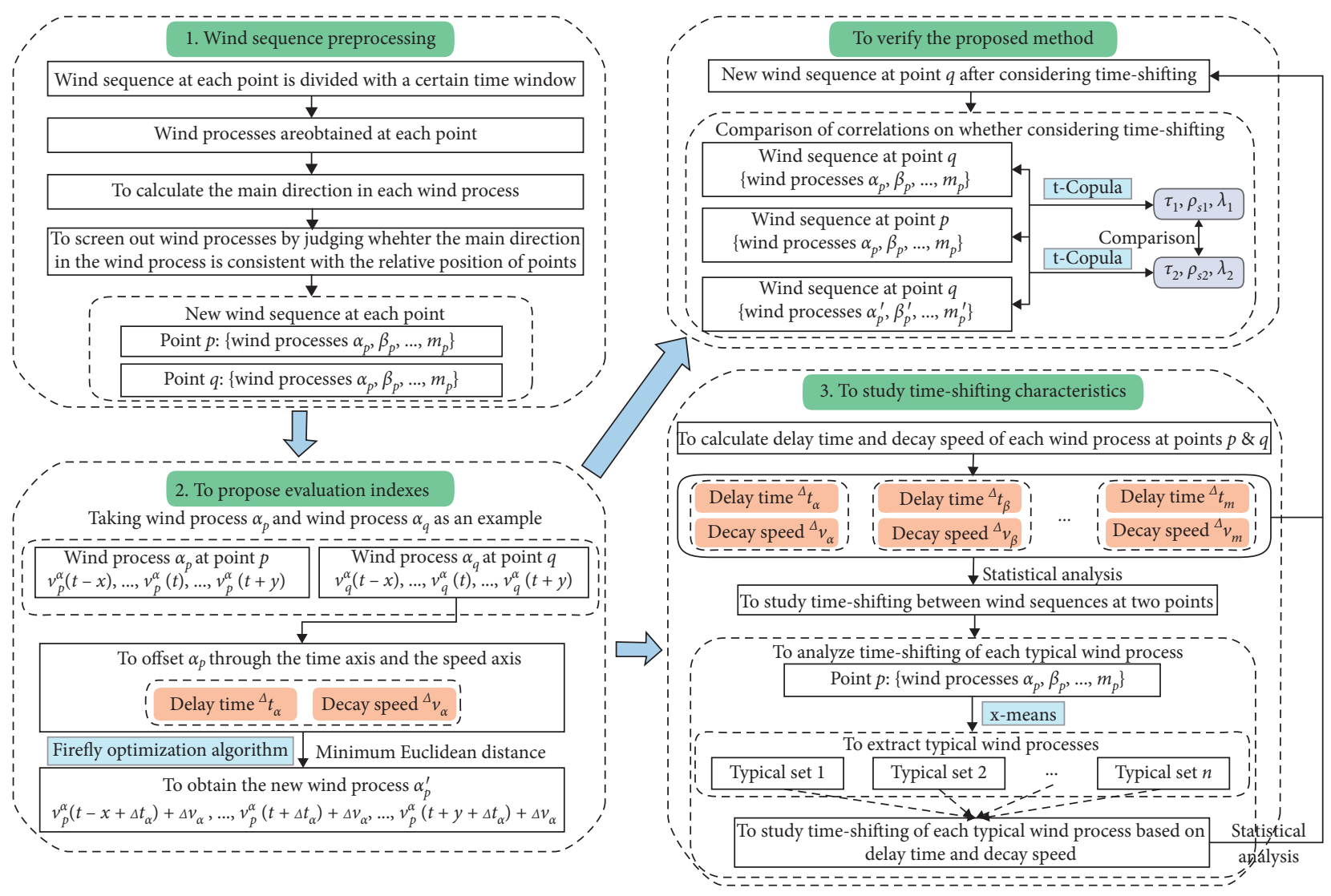

Figure 1: Outline of the research process on wind speed time-shifting.

contained when studying the time-shifting characteristics of wind speed sequences at different spatial positions. According to the research results of Risø Laboratory at the Technical University of Denmark, even for a large-scale wind farm of $30 \mathrm{~km} \times 30 \mathrm{~km}$ at the upwind direction, the weakening effect on the wind speed at the downwind direction could vanish after a distance of $30 \mathrm{~km}$ to $60 \mathrm{~km}$ [24]. Therefore, it can be considered that there is no influence of the decay speed among wind speed sequences at different locations when the distance between the wind farms exceeds $60 \mathrm{~km}$.

\subsection{Calculation Method of the Proposed Indexes Based on} Firefly Optimization Algorithm. The firefly optimization algorithm [25] is applied to calculate the proposed indexes, the delay time and decay speed. Each firefly is regarded as a point in the algorithm, and the fireflies are distributed randomly in the search space at first. There are two parameters in the firefly optimization algorithm, the brightness and the attractiveness. The brightness of the firefly is decided by the objective function, which indicates the position and decides the next moving direction. The attractiveness between two fireflies is inversely proportional to the distance, which decides the next moving distance. The firefly is attracted and moves toward the relatively brighter one according to the brightness and attractiveness; then the fireflies gather through the movement. In the optimization process, the brightness and attractiveness are updated iteratively until the optimization objective is achieved. The specific steps of the firefly algorithm are as follows.

Step (a): to set the initial parameters of the algorithm, including the maximum attraction $\beta 0$, the light attraction coefficient $\gamma$, the step factor $\alpha$, the maximum number of iterations, and the number of fireflies.

Step (b): to initialize the position of fireflies.

Step (c): to calculate the objective function of each firefly and regard it as the maximum fluorescent brightness $I_{0}$.

Step (d): to define the relative brightness between fireflies, as shown in the following equation:

$$
I=I_{0} e^{-\gamma r_{i j}},
$$

where $r_{i j}$ is the relative distance between firefly $i$ and firefly $j$ and the light attraction coefficient $\gamma$ reflects the weakening characteristics with the increase of the distance and the absorption of the propagation medium. to define the attractiveness between fireflies, as shown in the following equation:

$$
\beta=\beta_{0} e^{-\gamma r_{i j}^{2}}
$$

to calculate the relative brightness and attractiveness between fireflies according to equations (1) and (2) and sort them by the relative brightness. 
Step (e): to update the position of fireflies, as shown in the following equation:

$$
x_{i}=x_{i}+\beta\left(x_{j}-x_{i}\right)+\alpha \varepsilon_{i},
$$

where $x_{i}$ and $x_{j}$ are the positions of firefly $i$ and firefly $j$, respectively. The step factor $\alpha$ is a disturbance term, which is added to enlarge the search area and avoid the optimization algorithm falling into partial convergence prematurely. In general, $\alpha \in[0,1]$ and $\varepsilon_{i} \in[-0.5,0.5]$.

Step (f): to repeat Step (c) to Step (e) until the maximum iterations number is reached, and then the optimal results can be obtained.

2.3. Correlation Analysis Based on t-Copula Function. The correlation analysis between wind speed time series at different spatial points is an important method to test the validity of the proposed time-shifting indexes and evaluate the effect of the time-shifting characteristics. At present, most researchers use the linear correlation coefficient to measure the correlation relationship between wind speed sequences. However, when the distribution of the variable follows the nonnormal distribution or nonelliptic distribution, it may get wrong conclusions [26]. The distribution of wind speed is more consistent with Weibull distribution, so it is more reasonable to use the rank correlation coefficient to measure the correlation relationship between wind speed sequences. Furthermore, compared with the normal weather conditions, the wind power output in the extreme wind conditions has a greater impact on the electric power system, and the output correlation among wind turbines or wind farms should be paid more attention in these weather conditions.

$\mathrm{t}$-Copula [27] function is one of the commonly used Copula functions. It has a thicker tail and is more sensitive to the changes of the tail correlation between random variables, which can capture the symmetric tail correlation. Therefore, the $\mathrm{t}$-Copula function is used to fit the joint probability density of wind speed sequences under two conditions in this paper, including the original sequences and the sequences considering time-shifting characteristics. Then, the Kendall rank correlation coefficient, Spearman rank correlation coefficient, and tail correction coefficient can be calculated to verify the validity of the proposed time-shifting indexes and evaluate the effect of the time-shifting characteristics. The specific steps of the correlation analysis based on t-Copula function are as follows.

The binary t-Copula function is shown in equation (4), where the linear correlation coefficient between two variables is $\rho$ and the degree of freedom is $k$. The density function and the distribution function of $t$-Copula are depicted in Figure 2:

$$
\begin{aligned}
C^{t}(\mu, v ; \rho, k)= & \int_{-\infty}^{t_{k}^{-1}(\mu)} \int_{-\infty}^{t_{k}^{-1}(\nu)} \frac{1}{2 \pi \sqrt{1-\rho^{2}}} \\
& \cdot\left[1+\frac{s^{2}-2 \rho s t+t^{2}}{k\left(1-\rho^{2}\right)}\right]^{-(k+2) / 2} \mathrm{~d} s \mathrm{~d} t,
\end{aligned}
$$

where $t_{k}^{-1}$ is the inverse function of the distribution function of unary $t$ distribution with freedom degree of $k$.

The calculation equations of Kendall rank correlation coefficient $\tau$, Spearman rank correlation coefficient $\rho_{s}$, and tail correlation coefficient $\lambda$ based on $\mathrm{t}$-Copula function are shown in the following equations:

$$
\begin{aligned}
\tau & =4 \int_{0}^{1} \int_{0}^{1} C^{t}(\mu, \nu) \mathrm{d} C^{t}(\mu, \nu)-1 \\
& =\frac{2 \arcsin \rho}{\pi} \\
\rho_{s} & =12 \int_{0}^{1} \int_{0}^{1} C^{t}(\mu, \nu) \mathrm{d} \mu \mathrm{d} \nu-3 \\
& =\frac{6 \arcsin (\rho / 2)}{\pi}, \\
\lambda & =\lim _{\mu \longrightarrow 1^{-}} \frac{1-2 \mu+C^{t}(\mu, \mu)}{1-\mu} \\
& =\lim _{\mu \longrightarrow 0^{+}} \frac{C^{t}(\mu, \mu)}{\mu} \\
& =2-2 t_{k+1}\left(\frac{\sqrt{k+1} \sqrt{1-\rho}}{\sqrt{1+\rho}}\right),
\end{aligned}
$$

where $t_{k+1}((\sqrt{k+1} \sqrt{1-\rho}) / \sqrt{1+\rho})$ is the value of the distribution function of unary $t$ with freedom degree of $k+1$ at $(\sqrt{k+1} \sqrt{1-\rho}) / \sqrt{1+\rho}$.

\subsection{Extraction of Typical Wind Processes Based on the x-Means} Clustering Method. The improved algorithm of $k$-means algorithm, $x$-means [28], is adopted to extract the typical wind processes when analyzing the time-shifting characteristics of each typical wind process. Compared with $k$ means algorithm, $x$-means algorithm is not required to give the specific value of the clustering number $n$ in advance but only needs to give a range within which the true $n$ reasonably lies. The clustering result is not only the set of centroids but also the value for $n$ which scores best in this range. The specific steps of $x$-means are as follows.

Step (a): to input $m$ wind processes to be clustered and set the range of clustering number as $\left[n_{\min }, n_{\max }\right]$, where $n_{\max } \leq m$.

Step (b): to select $n_{\text {min }}$ initial clustering centers from original wind processes randomly.

Step (c): to calculate the Euclidean distance between other wind processes and each clustering center and assign the corresponding wind process to the nearest category.

Step (d): to calculate the average value of wind processes in each clustering set and use the results as the new clustering centers.

Step (e): to repeat Step (c) and Step (d) until the clustering centers no longer change, and the optimal clustering results can be obtained with the clustering number of $n_{\min }$. 


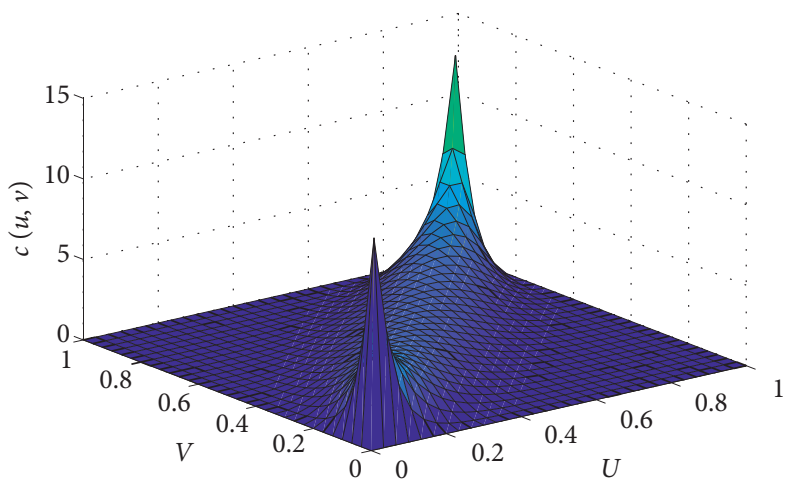

(a)

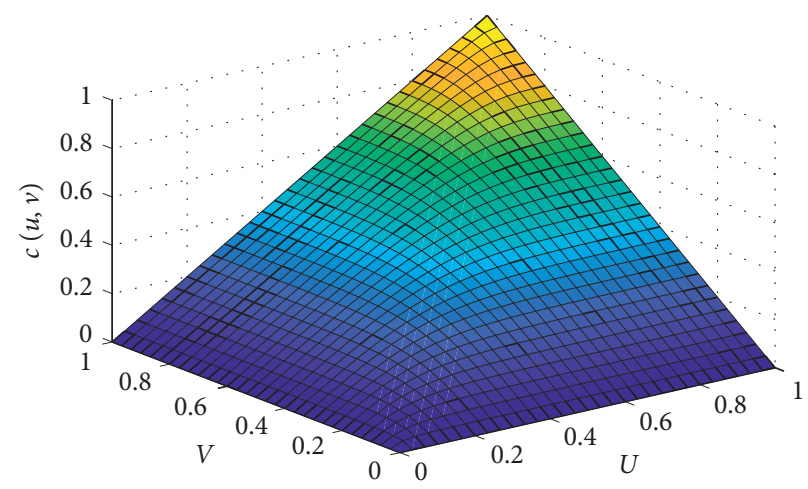

(b)

Figure 2: The density function and distribution function of binary t-Copula. (a) Density function. (b) Distribution function.

Step (b) to Step (e) are the main steps of $k$-means algorithm when the clustering number is $n_{\text {min }}$.

Step (f): to apply the 2-means algorithm in each clustering set, and the clustering results are evaluated according to the Bayesian Information Criterion (BIC). The calculation method of BIC can be seen in [28]. If the BIC score is larger when 2-means algorithm is used in the clustering set, the set will be divided into two sets; otherwise, the clustering set will be retained.

Step (g): to repeat Step (f) in each new clustering set until the clustering number $n$ is no longer increased or $n \geq n_{\text {max }}$.

\section{Case Study and Analysis}

This section analyzes the time-shifting characteristics of wind speed time series in different points under the wind farms scale and the wind turbines scale, respectively. The data includes wind speed and wind direction. In the wind farms scale, the data is the mesoscale data located in South Gobi of Mongolian; the time range is from January 1, 2004, to October 31, 2018, and the temporal resolution is $1 \mathrm{~h}$. All points are located in the flat terrain in this case. In the wind turbines scale, the data is the real operation data of one wind farm in Northern China; the time range is from January 1, 2014, to December 30, 2014, and the temporal resolution is $1 \mathrm{~min}$. All points are located in the hilly terrain in this case.

3.1. Time-Shifting under Wind Farms Scale. The wind sequence at each point is preprocessed at first. The wind sequence is divided into different wind processes with a time window of 1 day in this case, and then the wind process sets are obtained at each point. The relative distribution of each point is shown in Figure 3, so the wind processes with the main wind direction between $0265^{\circ}$ and $275^{\circ}$ at the corresponding point should be screened out for the time-shifting study. For example, when studying the time-shifting characteristics of wind speed sequences from point 1 to other points (2-5), the wind processes with the main direction between $265^{\circ}$ and $275^{\circ}$ at point 1 and the contemporaneous wind processes at other points should be screened out

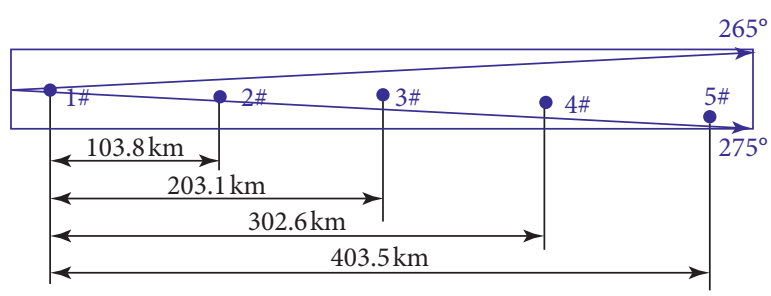

Figure 3: The relative distribution of points (wind farms scale).

simultaneously. Then, the new wind speed sequence that can be used to study the time-shifting characteristics at each point is reconstructed. The average speeds of new wind speed sequences at points 1 to 4 are $8.32 \mathrm{~m} / \mathrm{s}, 8.44 \mathrm{~m} / \mathrm{s}$, $6.68 \mathrm{~m} / \mathrm{s}$, and $6.06 \mathrm{~m} / \mathrm{s}$, respectively.

Then, the time-shifting characteristics of new wind speed sequences between point 1 and points 2 to 5 , between point 2 and points 3 to 5 , between point 3 and points 4 to 5 , and between point 4 and point 5 are studied, respectively. Since the distances between two points are all more than $100 \mathrm{~km}$, only the delay time is used as the time-shifting evaluation index in this case. The delay time distributions of wind speed time series between different points are shown in Figure 4. It can be seen that, with the increase of distance, the median delay time between wind speed sequences at two points increases, and the frequency distribution becomes more dispersed. The delay time of the wind speed sequence from point 1 to other points (2-5) varies in $0 \mathrm{~h}-26 \mathrm{~h}, 0 \mathrm{~h}-31 \mathrm{~h}$, $0 \mathrm{~h}-49 \mathrm{~h}$, and $0 \mathrm{~h}-78 \mathrm{~h}$, respectively. The average delay time between wind speed sequences at two points has a correlation relationship with the average wind speed at the starting point and the distance between two points, but it is not completely equal to the quotient between these two variables. Take the wind speed sequence at point 1 as an example; if the distances between point 1 and points 2 to 5 are divided by the average wind speed at point 1 , the corresponding results are $3 \mathrm{~h}, 7 \mathrm{~h}, 10 \mathrm{~h}$, and $13 \mathrm{~h}$, respectively. When the proposed time-shifting analysis method is applied, the average delay time of wind speed sequence from point 1 to other points $(2-5)$ is $2 \mathrm{~h}, 5 \mathrm{~h}, 8 \mathrm{~h}$, and $14 \mathrm{~h}$, respectively. This is because even if the average speed of two wind processes is the same, the wind conditions can also be quite 


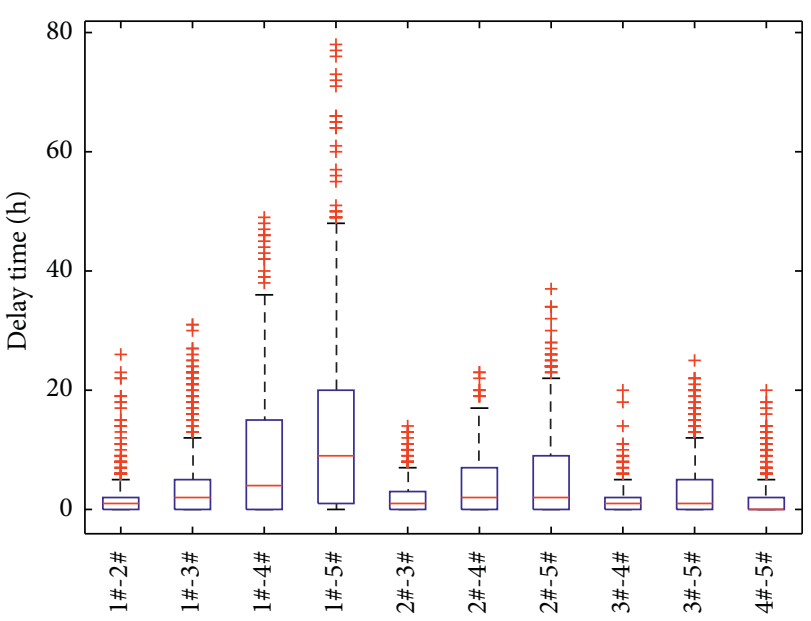

FIGURE 4: Delay time distributions of wind speed sequences between different points (wind farms scale).

different, and the time-shifting characteristics are various under different wind processes. Therefore, it is more reasonable to calculate the delay time between wind speed sequences at two points based on the similarity of wind processes.

In order to preliminarily analyze the mapping relationship among the time-shifting characteristics of wind speed sequences at different points, the distances between points, and the actual wind conditions, the wind processes at point 1 are divided according to the average wind speed of each process, with the interval of $1 \mathrm{~m} / \mathrm{s}$. The average delay time of point 1 to points $2-5$ is calculated in each wind speed bin, respectively, and the results are depicted in Figure 5. As can be seen, the delay time increases with the distance when the wind speed is almost the same and decreases with the wind speed when the distance is basically the same. However, since the wind speed is inevitably affected by various factors such as terrain and obstacles when it propagates from one point to another point in space, the propagation time is not equal to the quotient of distance and its value. Furthermore, the wind process is the time series composed of different wind speeds, so the propagation law of the wind process is more complicated. This is why some dots in Figure 5 look like the "abnormal dots," such as the average delay time from point 1 to point 2 when the average speed of the wind process is between $8 \mathrm{~m} / \mathrm{s}$ and $9 \mathrm{~m} / \mathrm{s}$ and the average delay time from point 1 to point 3 when the average speed of the wind process is between $11 \mathrm{~m} / \mathrm{s}$ and $12 \mathrm{~m} / \mathrm{s}$. Therefore, it is necessary to further analyze the time-shifting characteristics of different wind processes at different spatial scales.

$x$-Means algorithm is applied to cluster the wind processes at point 1 to extract typical wind processes, and then the typical wind process sets are constructed. The range of clustering number is set as in $[3,15]$ when using $x$-means algorithm. The optimal clustering number is 24 in this case. The average delay time of each wind process set from point 1 to other points $(2-5)$ is calculated, respectively, as shown in Figure 6. Six typical wind process sets are selected randomly, as depicted in Figure 7. The red solid line is the clustering center and the black-dashed lines are the actual wind

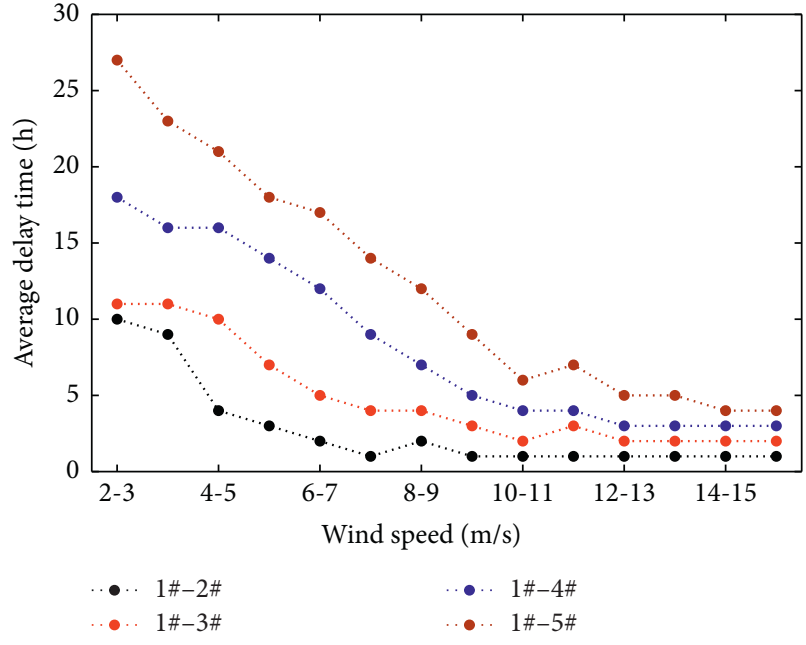

FIGURE 5: Average delay time between different points in each wind speed bin (wind farms scale).

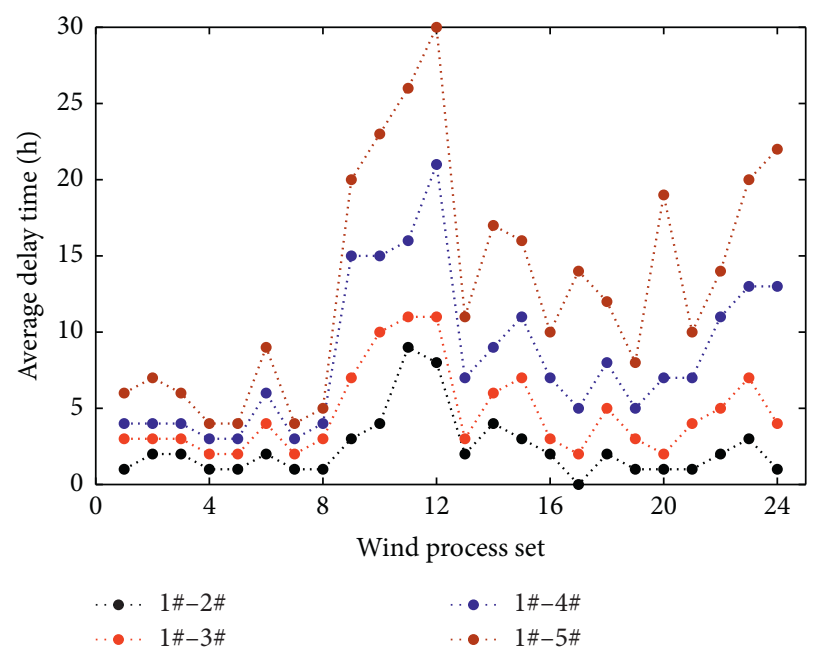

Figure 6: Average delay time in each wind process set between point 1 and other points (wind farms scale).

processes of each set. The average delay time from point 1 to other points and the average wind speed of these six typical wind process sets are listed in Table 1.

The new wind speed sequence at each point considering the time-shifting characteristics is reconstructed, respectively, according to the calculation results of the delay time. The wind speed time series of whether considering the timeshifting is shown in Figure 8. As can be seen, the wind speed sequences at different points could be more similar if the time-shifting characteristics are considered.

In order to further verify the validity of the proposed method, the correlation analysis is used to test the effectiveness of the time-shifting characteristics. t-Copula function is used to fit the original wind speed sequences of two points and the new wind speed sequences considering the time-shifting characteristics of two points, respectively. Then the Kendall correlation coefficient $\tau$, Spearman correlation coefficient $\rho_{s}$, and tail correlation coefficient $\lambda$ are 


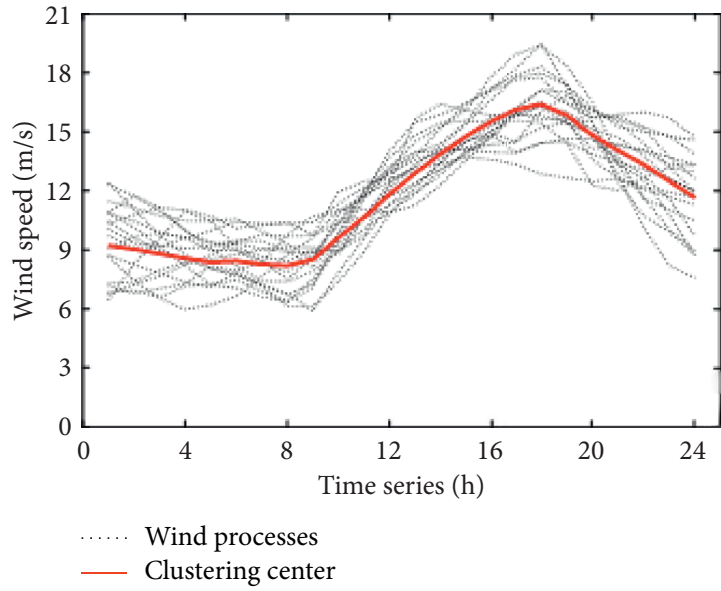

(a)

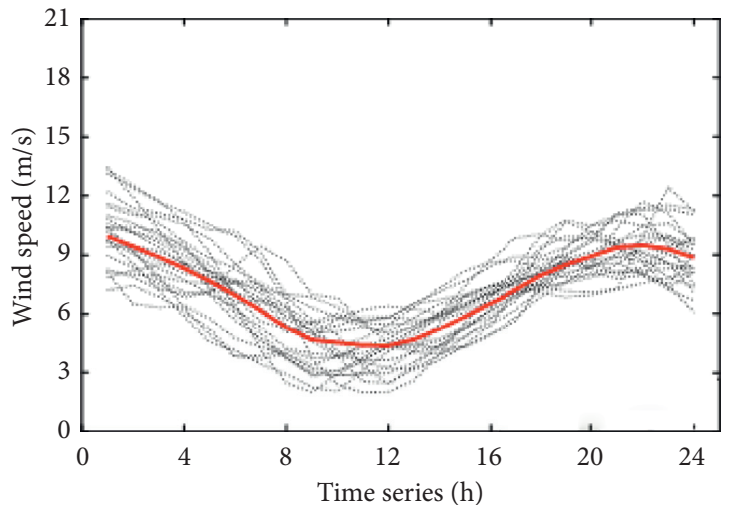

Wind processes

_- Clustering center

(c)

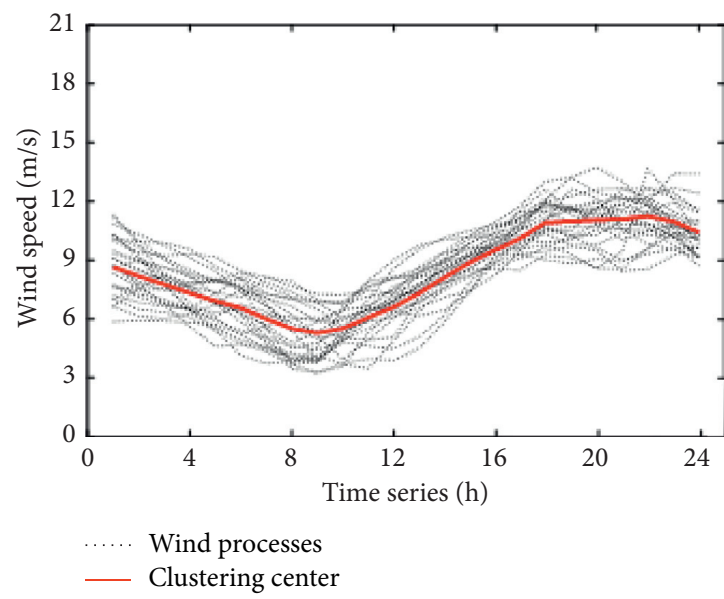

(e)

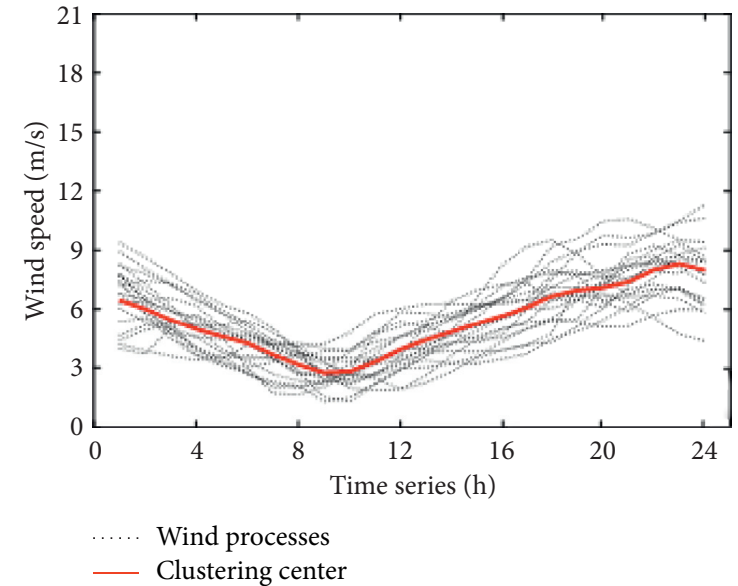

(b)

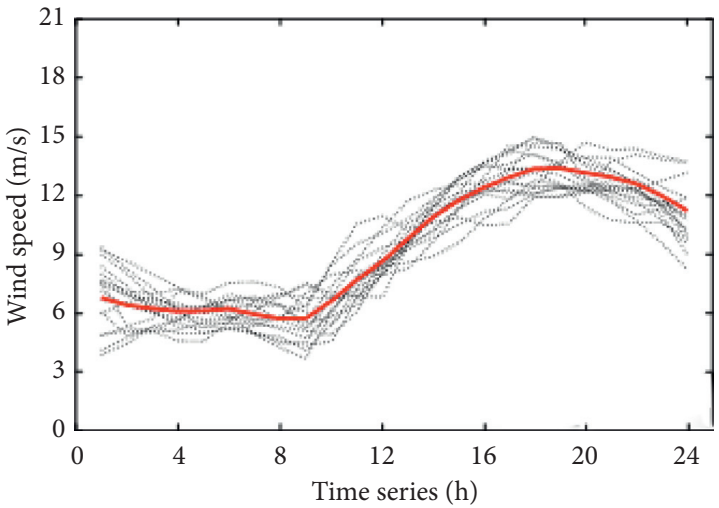

...... Wind processes

(d)

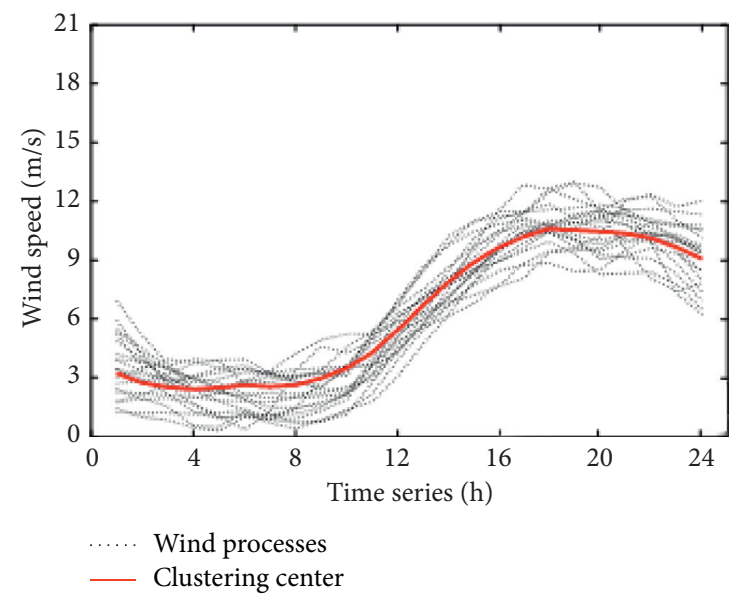

(f)

Figure 7: Six typical wind process sets at point 1 (wind farms scale). (a) Typical wind process set 6. (b) Typical wind process set 10. (c) Typical wind process set 15. (d) Typical wind process set 18. (e) Typical wind process set 22. (f) Typical wind process set 23.

calculated based on the fitted function, and the results are listed in Table 2. It can be seen that the correlation of wind speed sequences at different points is enhanced significantly when the time-shifting characteristics are considered. Since all points are located in the flat terrain in this case, the effect of time-shifting becomes more obvious as the distance increases. For example, taking point 1 as the starting point, if the time-shifting characteristics are considered, the correlations of wind speed sequences between point 1 and points 2 to 5 are increased by $0.048,0.097,0.123$, and 0.227 , 
TABLe 1: Average delay time in six wind process sets between point 1 and other points (wind farms scale).

\begin{tabular}{lccccc}
\hline \multirow{2}{*}{ Typical wind process set } & \multicolumn{2}{c}{ Average delay time (h) } & Average wind speed (m/s) \\
\hline 6 & $1 \#-2 \#$ & $1 \#-3 \#$ & $1 \#-4 \#$ & 6 & 9 \\
11.84 \\
10 & 2 & 4 & 15 & 23 & 5.54 \\
18 & 4 & 10 & 11 & 16 & 7.27 \\
22 & 3 & 7 & 8 & 12 & 9.49 \\
23 & 2 & 5 & 11 & 14 & 8.37 \\
\hline
\end{tabular}
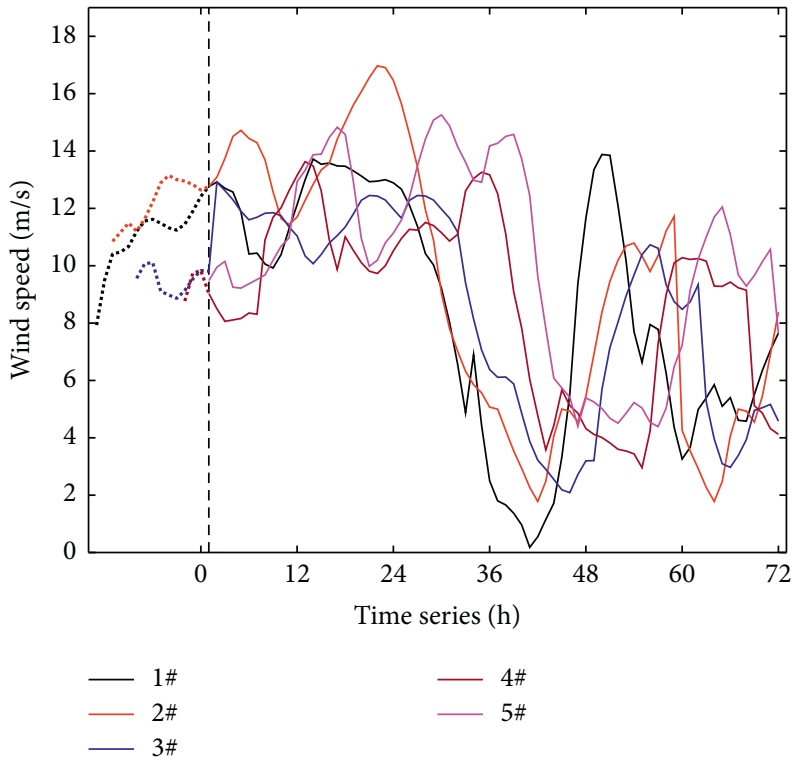

(a)

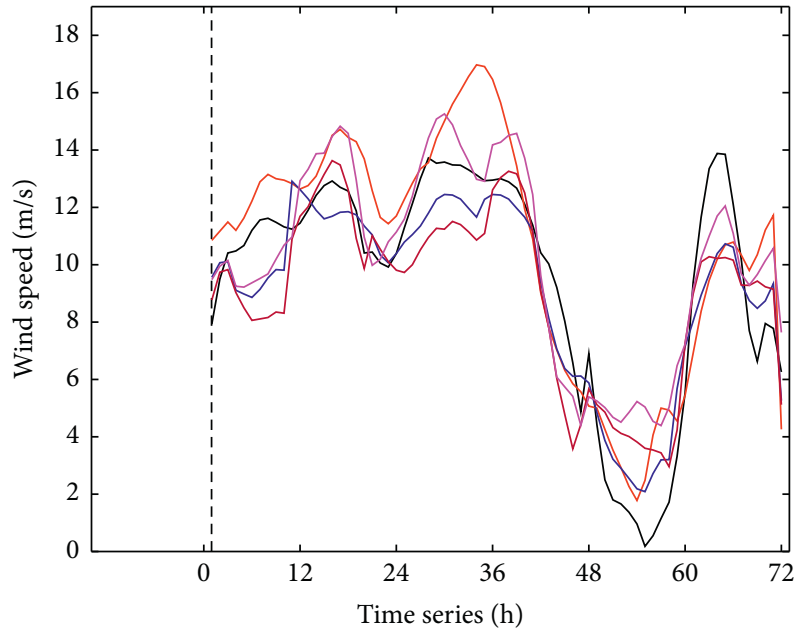

$1 \#$
$-\quad 2 \#$
$-3 \#$
$4 \#$
$-5 \#$

(b)

FIGURE 8: Wind speed sequences of whether considering time-shifting characteristics (wind farms scale). (a) Original. (b) Considering timeshifting.

TABLE 2: Correlation of wind speed sequences between points under two conditions (wind farms scale).

\begin{tabular}{lcccccccccc}
\hline$\tau$ & $1 \#-2 \#$ & $1 \#-3 \#$ & $1 \#-4 \#$ & $1 \#-5 \#$ & $2 \#-3 \#$ & $2 \#-4 \#$ & $2 \#-5 \#$ & $3 \#-4 \#$ & $3 \#-5 \#$ & $4 \#-5 \#$ \\
\hline Original & 0.569 & 0.479 & 0.399 & 0.283 & 0.641 & 0.485 & 0.464 & 0.681 & 0.555 & 0.615 \\
Considering time-shifting & 0.616 & 0.577 & 0.522 & 0.510 & 0.655 & 0.543 & 0.567 & 0.712 & 0.611 & 0.648 \\
Difference & $\mathbf{0 . 0 4 8}$ & $\mathbf{0 . 0 9 7}$ & $\mathbf{0 . 1 2 3}$ & $\mathbf{0 . 2 2 7}$ & $\mathbf{0 . 0 1 4}$ & $\mathbf{0 . 0 5 8}$ & $\mathbf{0 . 1 0 3}$ & $\mathbf{0 . 0 3 0}$ & $\mathbf{0 . 0 5 6}$ & $\mathbf{0 . 0 3 3}$ \\
\hline$\rho_{s}$ & $1 \#-2 \#$ & $1 \#-3 \#$ & $1 \#-4 \#$ & $1 \#-5 \#$ & $2 \#-3 \#$ & $2 \#-4 \#$ & $2 \#-5 \#$ & $3 \#-4 \#$ & $3 \#-5 \#$ & $4 \#-5 \#$ \\
\hline Original & 0.756 & 0.660 & 0.563 & 0.411 & 0.831 & 0.670 & 0.640 & 0.862 & 0.743 & 0.805 \\
Considering time-shifting & 0.802 & 0.767 & 0.712 & 0.699 & 0.845 & 0.735 & 0.758 & 0.886 & 0.801 & 0.834 \\
Difference & $\mathbf{0 . 0 4 6}$ & $\mathbf{0 . 1 0 7}$ & $\mathbf{0 . 1 4 9}$ & $\mathbf{0 . 2 8 8}$ & $\mathbf{0 . 0 1 4}$ & $\mathbf{0 . 0 6 5}$ & $\mathbf{0 . 1 1 8}$ & $\mathbf{0 . 0 2 4}$ & $\mathbf{0 . 0 5 8}$ & $\mathbf{0 . 0 2 9}$ \\
\hline$\lambda$ & $1 \#-2 \#$ & $1 \#-3 \#$ & $1 \#-4 \#$ & $1 \#-5 \#$ & $2 \#-3 \#$ & $2 \#-4 \#$ & $2 \#-5 \#$ & $3 \#-4 \#$ & $3 \#-5 \#$ & $4 \#-5 \#$ \\
\hline Original & 0.348 & 0.226 & 0.093 & 0.040 & 0.095 & 0.106 & 0.241 & 0.463 & 0.318 & 0.301 \\
Considering time-shifting & 0.438 & 0.276 & 0.118 & 0.085 & 0.138 & 0.119 & 0.244 & 0.507 & 0.333 & 0.390 \\
Difference & $\mathbf{0 . 0 9 0}$ & $\mathbf{0 . 0 5 1}$ & $\mathbf{0 . 0 2 5}$ & $\mathbf{0 . 0 4 5}$ & $\mathbf{0 . 0 4 3}$ & $\mathbf{0 . 0 1 3}$ & $\mathbf{0 . 0 0 3}$ & $\mathbf{0 . 0 4 4}$ & $\mathbf{0 . 0 1 5}$ & $\mathbf{0 . 0 8 9}$ \\
\hline
\end{tabular}

respectively, when the Kendall correlation coefficient $\tau$ is used as the evaluation index; the correlations are increased by $0.046,0.107,0.149$, and 0.288 , respectively, when the Spearman correlation coefficient $\rho_{s}$ is used as the evaluation index. Furthermore, the tail correlation of wind speed sequences is also improved when the time-shifting characteristics are taken into account, which means the appearance times under high wind conditions and small wind conditions are more consistent. The tail correlation coefficients of wind speed sequences between point 1 and points 2 to 5 are increased by $0.090,0.050,0.025$, and 0.045 , respectively. 


\subsection{Time-Shifting under Wind Turbines Scale Containing} Wake Effect. The wind sequence at each point is preprocessed at first. The wind sequence is divided into different wind processes with a time window of 1 hour in this case, and then the wind process sets are obtained at each point. The relative distribution of each point is shown in Figure 9, so the wind processes with the main wind direction between $130^{\circ}$ and $140^{\circ}$ at the corresponding point should be screened out for the time-shifting study. The average speeds of new wind speed sequences at points 1 to 3 are $11.45 \mathrm{~m} / \mathrm{s}, 10.17 \mathrm{~m} /$ $\mathrm{s}$, and $10.49 \mathrm{~m} / \mathrm{s}$, respectively.

Then, the time-shifting characteristics of new wind speed sequences between point 1 and points 2 to 4 , between point 2 and points 3 to 4 , and between point 3 and point 4 are studied, respectively. Since the data used in this case is the actual operation data of the wind farm, the factors that affect wind speed sequences at different locations should include both the delay time and the decay speed. The distributions of delay time and decay speed of wind speed time series between different points are shown in Figure 10. It can be seen that, with the increase of distance, the median delay time between wind speed sequences at two points increases, and the frequency distribution becomes more dispersed. The wind speed at the downstream location is not always lower than that at the upstream location. The average decay speeds of the wind speed sequence from point 1 to other points $(2-4)$ are $0.62 \mathrm{~m} / \mathrm{s},-0.16 \mathrm{~m} / \mathrm{s}$, and $0.87 \mathrm{~m} / \mathrm{s}$, respectively. This is because the points are all located in the hilly terrain in this case, and the propagation of wind speed between different points is greatly affected by the terrain. Furthermore, the wake effect of wind turbines is weakened with the increase of the distance and the relief of the terrain.

In order to preliminarily analyze the mapping relationship among the time-shifting characteristics of wind speed sequences at different points, the distances between points, and the actual wind conditions, the wind processes at point 1 are divided according to the average wind speed of each process, with the interval of $1 \mathrm{~m} / \mathrm{s}$. The average delay time and average decay speed of wind processes from point 1 to points 2-4 are calculated in each wind speed bin, respectively, and the results are depicted in Figure 11. As can be seen, the delay time increases with the distance when the wind speed is almost the same and decreases with the wind speed when the distance is basically the same. However, due to the influence of the small distance between two points and the low time resolution of the data, the delay time of wind speed sequence between two points does not decrease with the increase of wind speed in some situations. This situation happens more frequently with the shortening of the distance between two points. The decay speed of wind speed sequences between different points varies with the value of wind speed.

The improved algorithm of $k$-means, $x$-means, is employed to cluster the wind processes at point 1 to extract typical wind processes, and then the typical wind process sets are constructed. The range of clustering number is set as in $[4,20]$, and the optimal clustering number is 32 in this case. The average delay time and the average decay speed of each wind process set from point 1 to other points (2-4) are calculated, respectively, and the results are shown in Figure 12. Six typical wind process

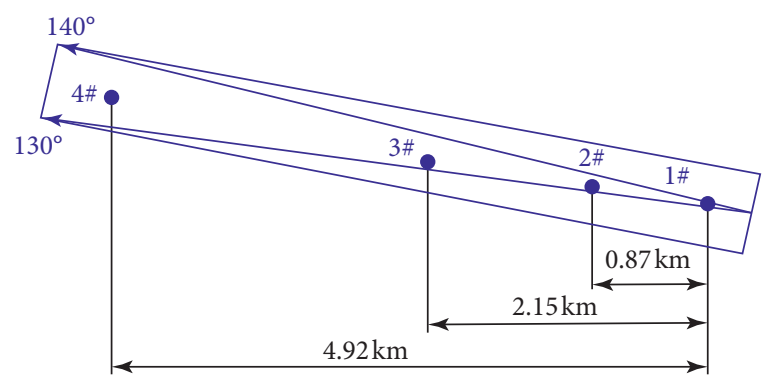

FIgURE 9: The relative distribution of points (wind turbines scale).

sets are selected randomly, as depicted in Figure 13. The average delay time and the average decay speed from point 1 to other points and the average wind speeds of these six typical wind process sets are listed in Table 3.

The new wind speed sequence at each point considering the time-shifting characteristics is reconstructed, respectively, according to the calculation results of the delay time and decay speed. The wind speed time series of whether considering the time-shifting is shown in Figure 14. As can be seen, the wind speed sequences at different points could be more similar if the time-shifting characteristics are considered.

In order to further verify the validity of the proposed method, the correlation analysis is used to test the effectiveness of the time-shifting characteristics. t-Copula function is used to fit the original wind speed sequences of two points and the new wind speed sequences considering the timeshifting characteristics of two points, respectively. Then the Kendall correlation coefficient $\tau$, Spearman correlation coefficient $\rho_{s}$, and tail correlation coefficient $\lambda$ are calculated based on the fitted function, and the results are listed in Table 4. It can be seen that the correlation of wind speed sequences at different points is enhanced significantly when the time-shifting characteristics are considered. For example, taking point 1 as the starting point, if the time-shifting characteristics are taken into account, the correlations of wind speed time series between point 1 and points 2 to 4 are increased by $0.142,0.103$, and 0.202 , respectively, when the Kendall correlation coefficient $\tau$ is used as the evaluation index; the correlations are increased by $0.065,0.046$, and 0.124 , respectively, when the Spearman correlation coefficient $\rho_{s}$ is used as the evaluation index. Furthermore, the tail correlations of wind speed sequences between point 1 and points 2 to 4 are increased by $0.374,0.147$, and 0.802 , respectively. It should be noted that, different from the case in wind farms scale, the points are all located in the hilly terrain in this case, the mapping relationship among the wind speed sequences at different points is more complicated, and the time-shifting effect does not show a more obvious trend with the increase of distance.

\section{Verification of the Proposed Time-Shifting Evaluation Method}

This section takes the data on December 31, 2014, under the wind turbines scale as an example to verify the calculation results of time-shifting and illustrate the effectiveness of the 


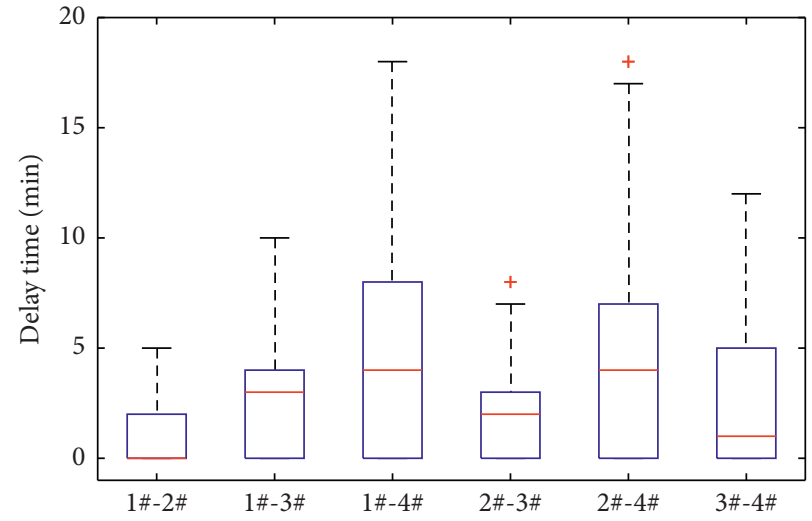

(a)

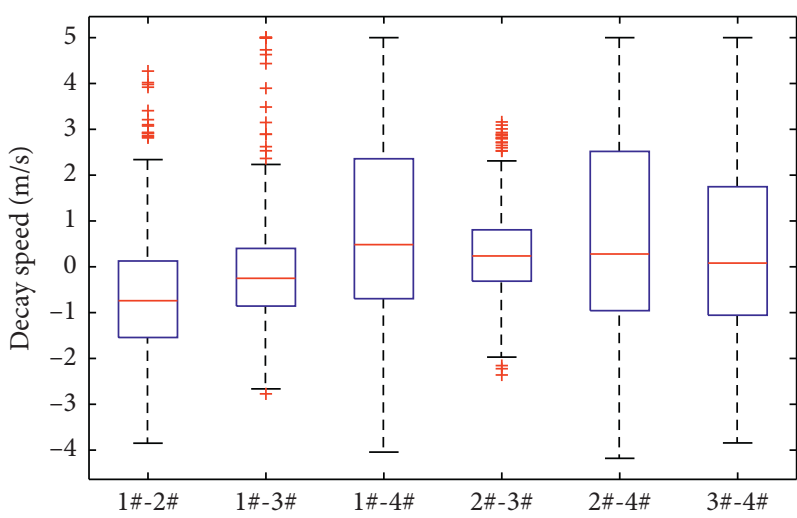

(b)

FIGURE 10: The time-shifting distributions of wind speed sequences between different points (wind turbines scale). (a) Delay time. (b) Decay speed.

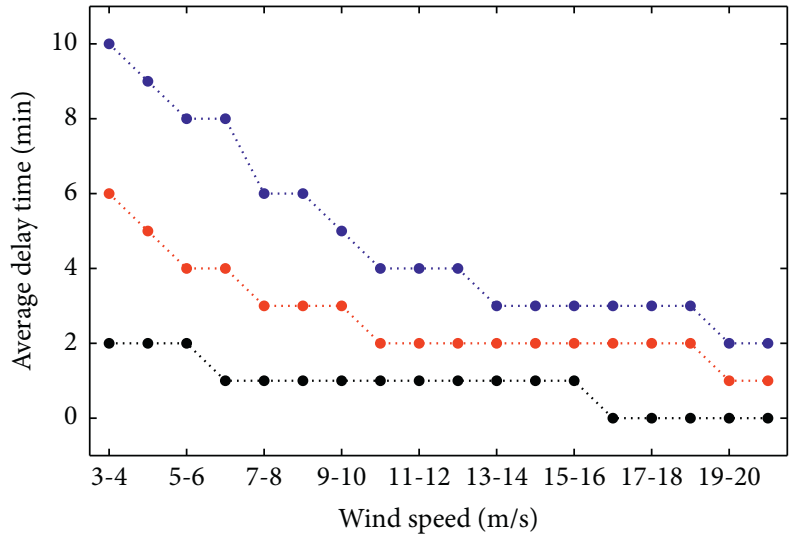

-. $1 \#-2 \#$

-.. $1 \#-3 \#$

.. $1 \#-4 \#$

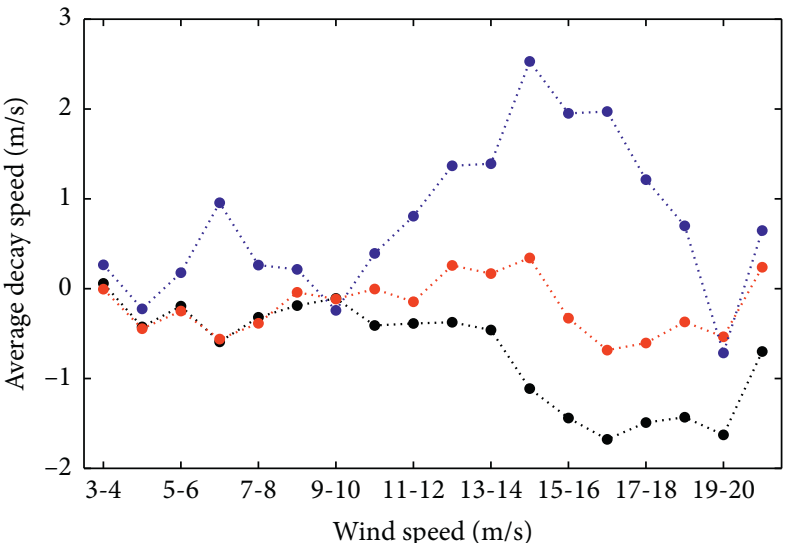

-.. 1\#-2\#

-.. $1 \#-3 \#$

-.. 1\#-4\#

(a)

(b)

FIGURE 11: The time-shifting characteristics between different points in each wind speed bin (wind turbines scale). (a) Average delay time. (b) Average decay speed.

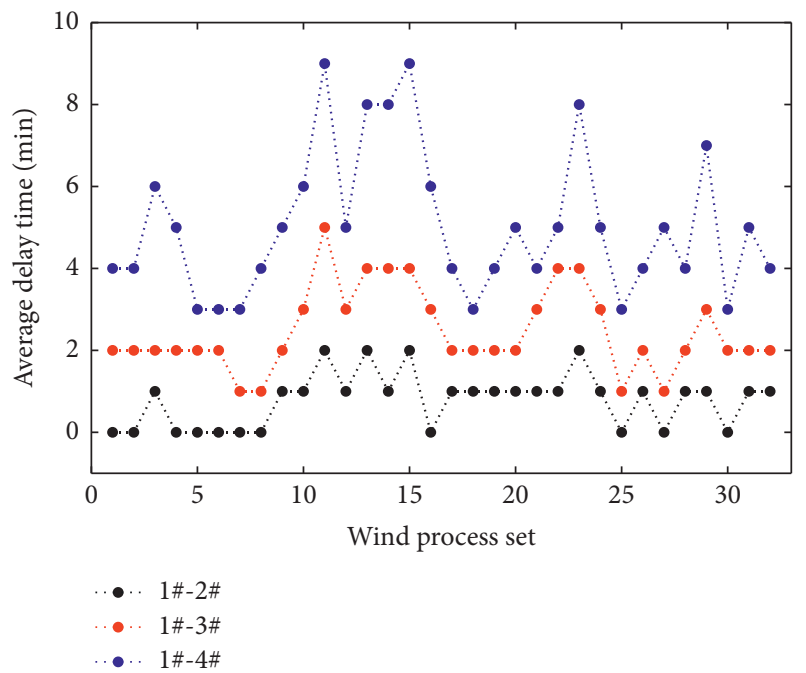

(a)

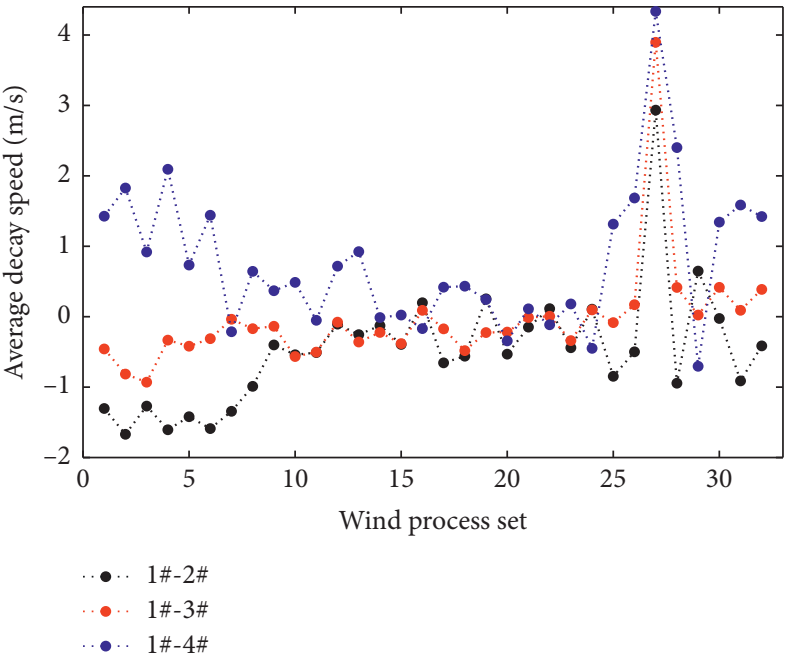

(b)

Figure 12: The time-shifting characteristics in each wind process set between point 1 and other points (wind turbines scale). (a) Average delay time. (b) Average decay speed. 


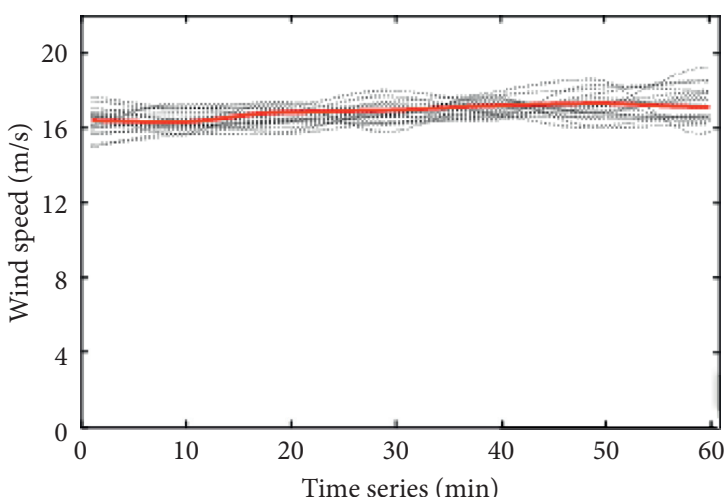

.... Wind processes

_ Clustering center

(a)

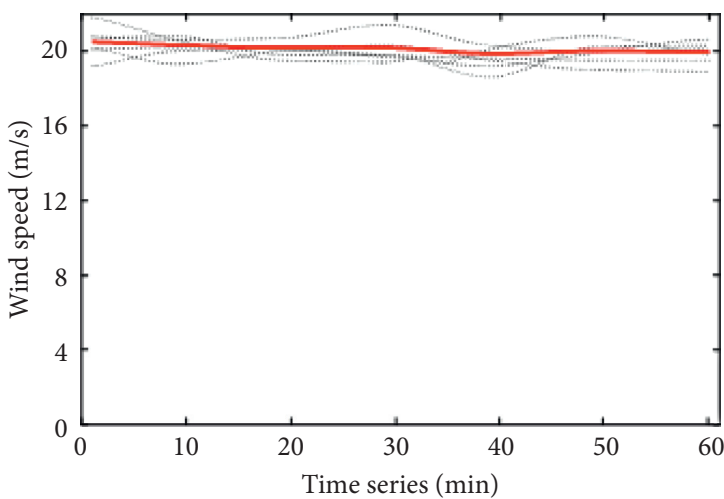

..... Wind processes

_ Clustering center

(c)

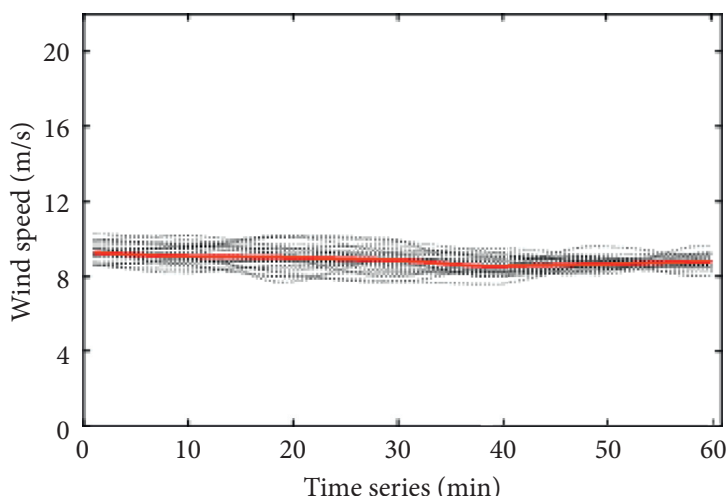

Wind processes

Clustering center

(e)

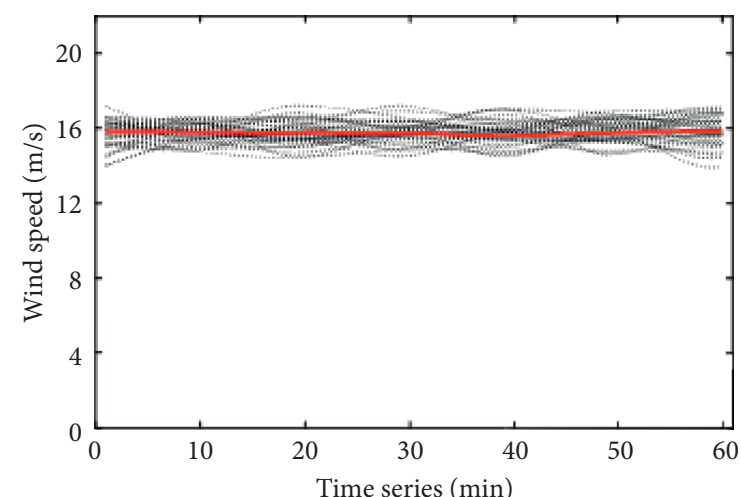

Wind processes

_ Clustering center

(b)

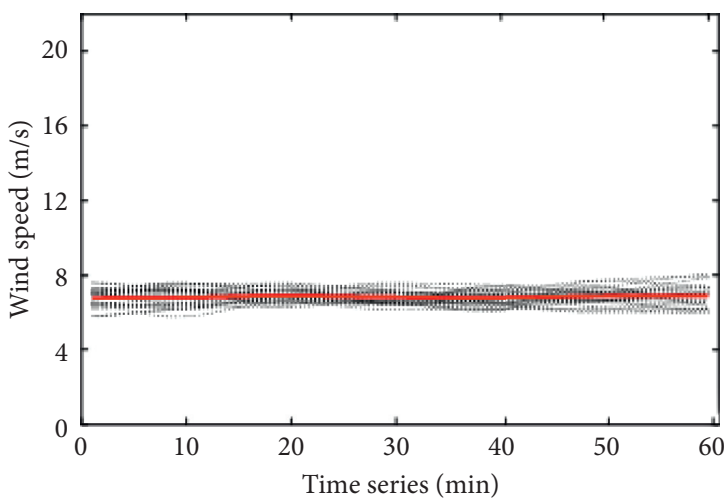

.... Wind processes

_ Clustering center

(d)

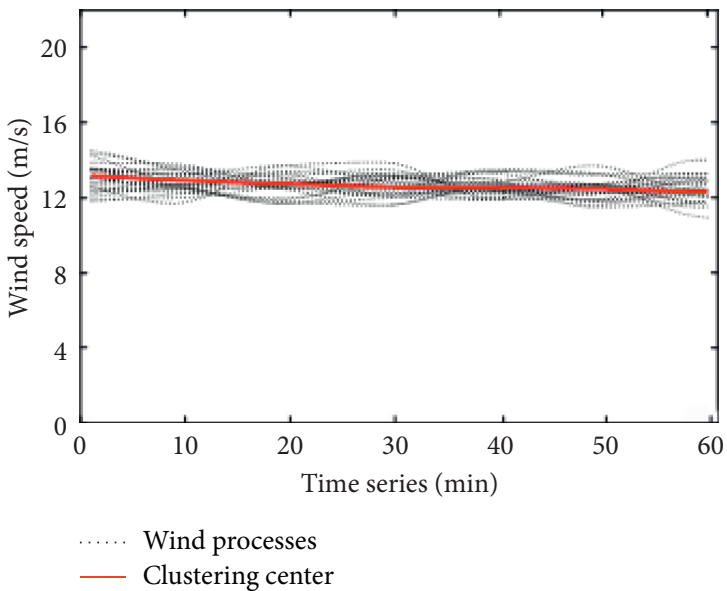

(f)

Figure 13: Six typical wind process sets at point 1 (wind turbines scale). (a) Typical wind process set 2. (b) Typical wind process set 4. (c) Typical wind process set 7. (d) Typical wind process set 11. (e) Typical wind process set 23. (f) Typical wind process set 31.

proposed evaluation method in this paper. The wind speed sequences at points 2 to 4 can be considered the same as that at point 1 if the time-shifting characteristics are not taken into account. When the time-shifting characteristics are considered, the wind speed sequences at points 2 to 4 should be calculated, respectively, based on the research results of time-shifting. The specific steps are as follows: first, to divide the wind speed sequence and the wind direction sequence at point 1 with a time window of 1 hour and, second, to judge whether the main wind direction at point 1 is consistent with the relative position of wind turbines in each wind process, that is, whether the main direction of the wind process at 
TABle 3: Average delay time and average decay speed in six wind process sets between point 1 and other points (wind turbines scale).

\begin{tabular}{|c|c|c|c|c|c|c|c|}
\hline \multirow{2}{*}{ Typical wind process set } & \multicolumn{3}{|c|}{ Average delay time (min) } & \multicolumn{3}{|c|}{ Average decay speed $(\mathrm{m} / \mathrm{s})$} & \multirow{2}{*}{ Average wind speed $(\mathrm{m} / \mathrm{s})$} \\
\hline & $1 \#-2 \#$ & $1 \#-3 \#$ & $1 \#-4 \#$ & $1 \#-2 \#$ & $1 \#-3 \#$ & $1 \#-4 \#$ & \\
\hline 2 & 0 & 2 & 4 & -1.67 & -0.81 & 1.83 & 16.89 \\
\hline 4 & 0 & 2 & 5 & -1.61 & -0.33 & 2.09 & 15.75 \\
\hline 7 & 0 & 1 & 3 & -1.34 & -0.04 & -0.21 & 20.12 \\
\hline 11 & 2 & 5 & 9 & -0.51 & -0.50 & -0.05 & 6.83 \\
\hline 23 & 2 & 4 & 8 & -0.44 & -0.34 & 0.18 & 8.81 \\
\hline 31 & 1 & 2 & 5 & -0.91 & 0.09 & 1.59 & 12.67 \\
\hline
\end{tabular}

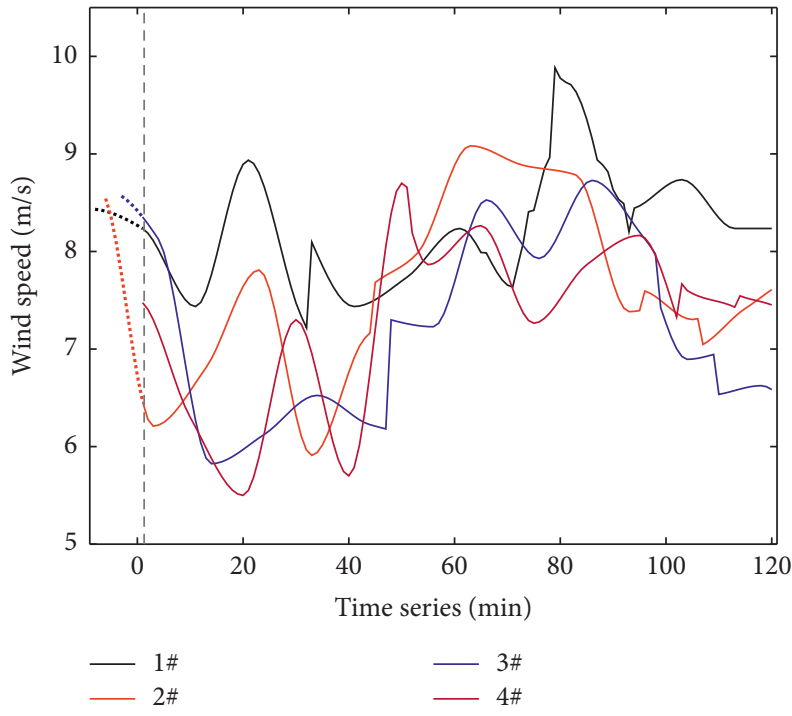

(a)

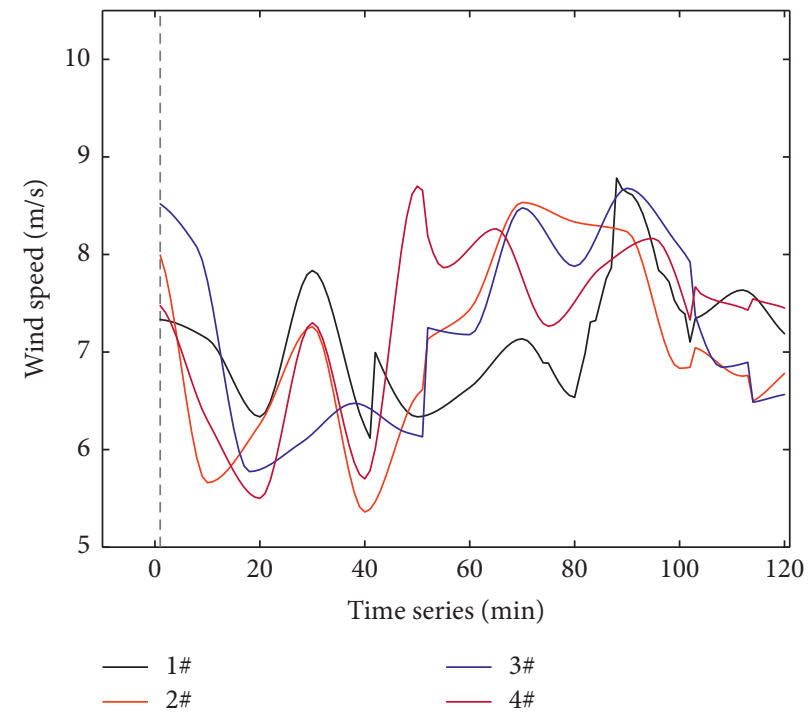

(b)

FIGURE 14: Wind speed sequences of whether considering time-shifting characteristics (wind turbines scale). (a) Original. (b) Considering time-shifting.

point 1 is between $130^{\circ}$ and $140^{\circ}$. If so, it should be judged at first which wind process set the wind process belongs to, and then the wind process at points 2 to 4 should be constructed according to the above calculation results of time-shifting. If not, the corresponding wind speed at points 2 to 4 can be considered the same as that at point 1 . The actual wind speed sequence and wind speed sequences of whether considering time-shifting at points 2 to 4 are shown in Figure 15. It can be seen that the wind speed sequence is more similar to the actual sequence at each point if the time-shifting characteristics are considered.

To further verify the calculation results of timeshifting, the average absolute deviations between the calculated wind speed sequences of whether considering the time-shifting and the actual wind speed sequence are used as the evaluation index to quantify the effectiveness of time-shifting. The average absolute deviations between the calculated wind speed sequence and the actual wind speed sequence at points 2 to 4 are $1.27 \mathrm{~m} / \mathrm{s}, 1.18 \mathrm{~m} / \mathrm{s}$, and $1.26 \mathrm{~m} / \mathrm{s}$, respectively, if the time-shifting is not taken into account. If the time-shifting is considered, the average absolute deviations are $1.06 \mathrm{~m} / \mathrm{s}, 1.02 \mathrm{~m} / \mathrm{s}$, and $1.04 \mathrm{~m} / \mathrm{s}$, respectively. The average absolute deviations are reduced by $16.5 \%, 13.6 \%$, and $17.5 \%$, respectively, when
TABlE 4: Correlation of wind speed sequences between points under two conditions (wind turbines scale).

\begin{tabular}{lcccccc}
\hline$\tau$ & $1 \#-2 \#$ & $1 \#-3 \#$ & $1 \#-4 \#$ & $2 \#-3 \#$ & $2 \#-4 \#$ & $3 \#-4 \#$ \\
\hline Original & 0.758 & 0.794 & 0.651 & 0.827 & 0.587 & 0.649 \\
$\begin{array}{l}\text { Considering time- } \\
\text { shifting }\end{array}$ & 0.900 & 0.897 & 0.853 & 0.912 & 0.853 & 0.872 \\
Difference & $\mathbf{0 . 1 4 2}$ & $\mathbf{0 . 1 0 3}$ & $\mathbf{0 . 2 0 2}$ & $\mathbf{0 . 0 8 6}$ & $\mathbf{0 . 2 6 6}$ & $\mathbf{0 . 2 2 3}$ \\
\hline$\rho_{s}$ & $1 \#-2 \#$ & $1 \#-3 \#$ & $1 \#-4 \#$ & $2 \#-3 \#$ & $2 \#-4 \#$ & $3 \#-4 \#$ \\
\hline Original & 0.920 & 0.938 & 0.842 & 0.959 & 0.783 & 0.839 \\
$\begin{array}{l}\text { Considering time- } \\
\text { shifting }\end{array}$ & 0.985 & 0.983 & 0.966 & 0.988 & 0.965 & 0.974 \\
$\begin{array}{l}\text { Difference } \\
\lambda\end{array}$ & $\mathbf{0 . 0 6 5}$ & $\mathbf{0 . 0 4 6}$ & $\mathbf{0 . 1 2 4}$ & $\mathbf{0 . 0 2 9}$ & $\mathbf{0 . 1 8 2}$ & $\mathbf{0 . 1 3 5}$ \\
\hline $\begin{array}{l}\text { Original } \\
\text { Considering time- } \\
\text { shifting }\end{array}$ & 0.467 & 0.703 & 0.004 & 0.517 & 0.000 & 0.058 \\
Difference & 0.841 & 0.850 & 0.806 & 0.872 & 0.828 & 0.813 \\
\hline
\end{tabular}

the time-shifting is considered. The average absolute deviations between wind speed sequences of whether considering the time-shifting and the actual wind speed sequence in each time window at points 2 to 4 are depicted in Figure 16. It can be seen that the wind speed 


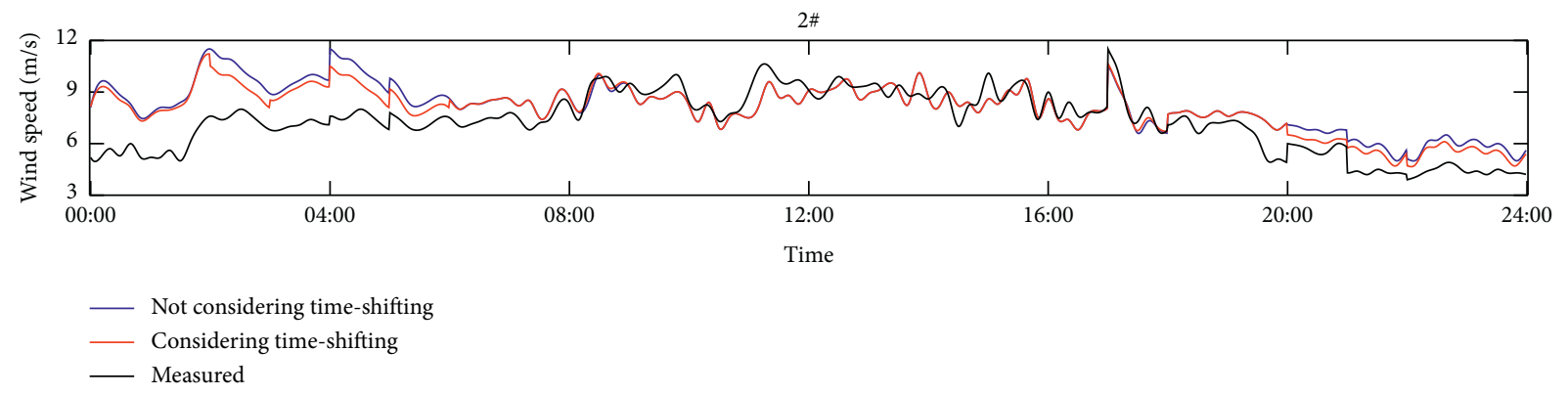

(a)

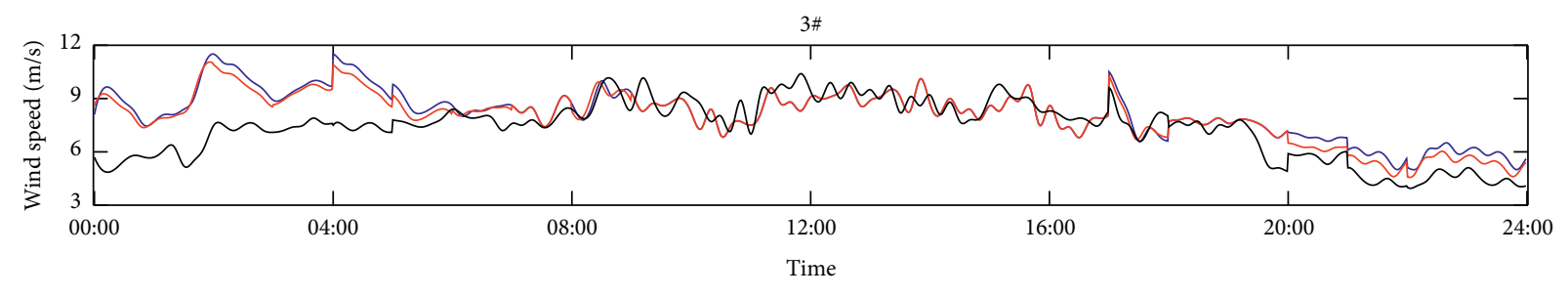

_ Not considering time-shifting

_ Considering time-shifting

- Measured

(b)

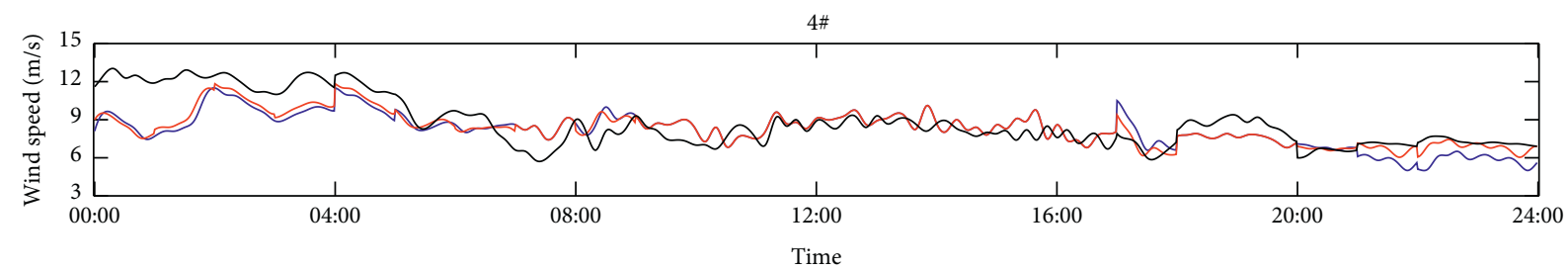

__ Not considering time-shifting

_ Considering time-shifting

- Measured

(c)

FIGURE 15: Wind speed sequences of whether considering the time-shifting characteristics at each point.

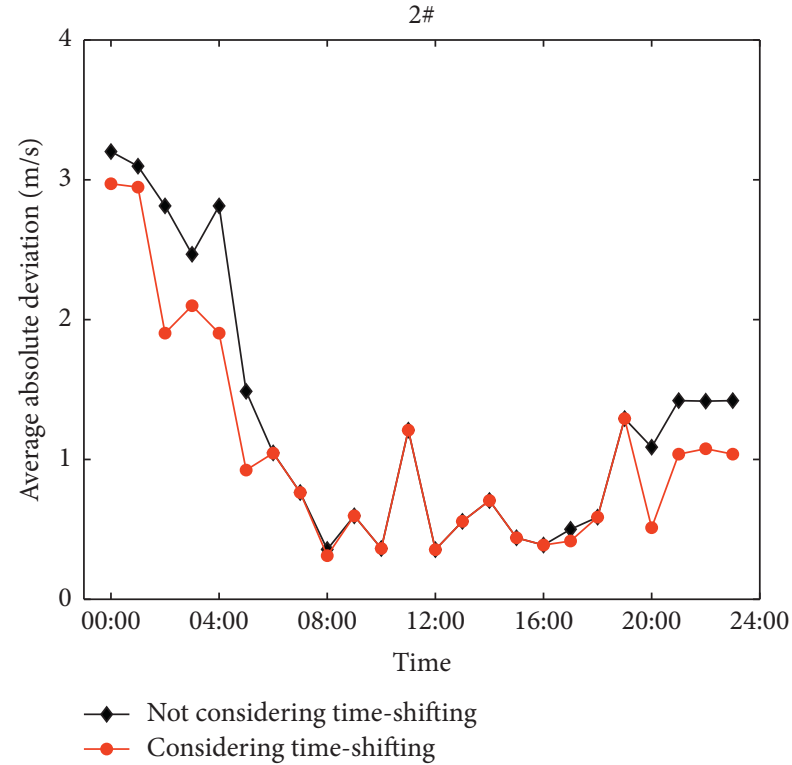

(a)

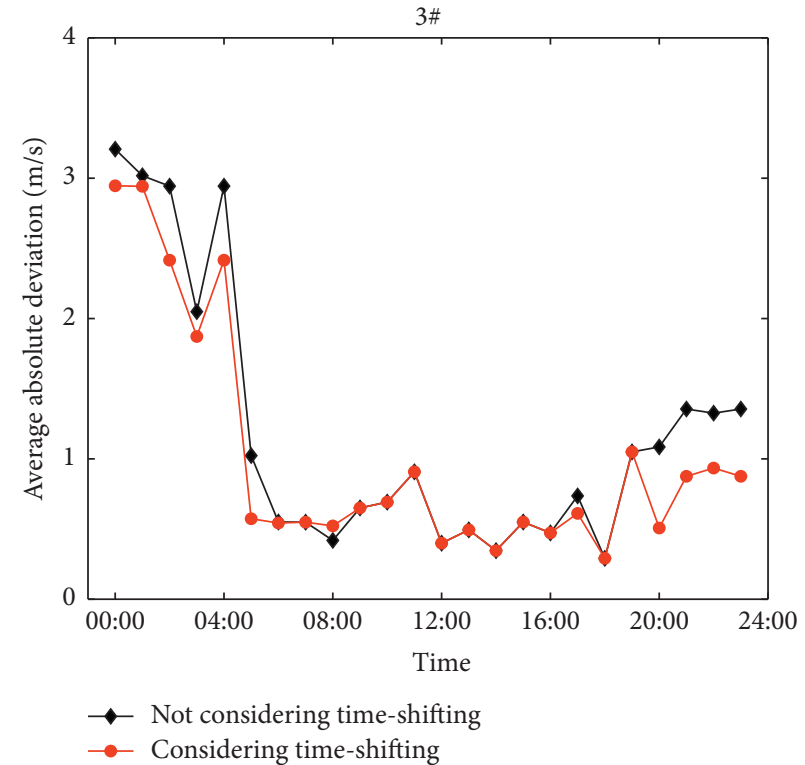

(b)

FIgURE 16: Continued. 


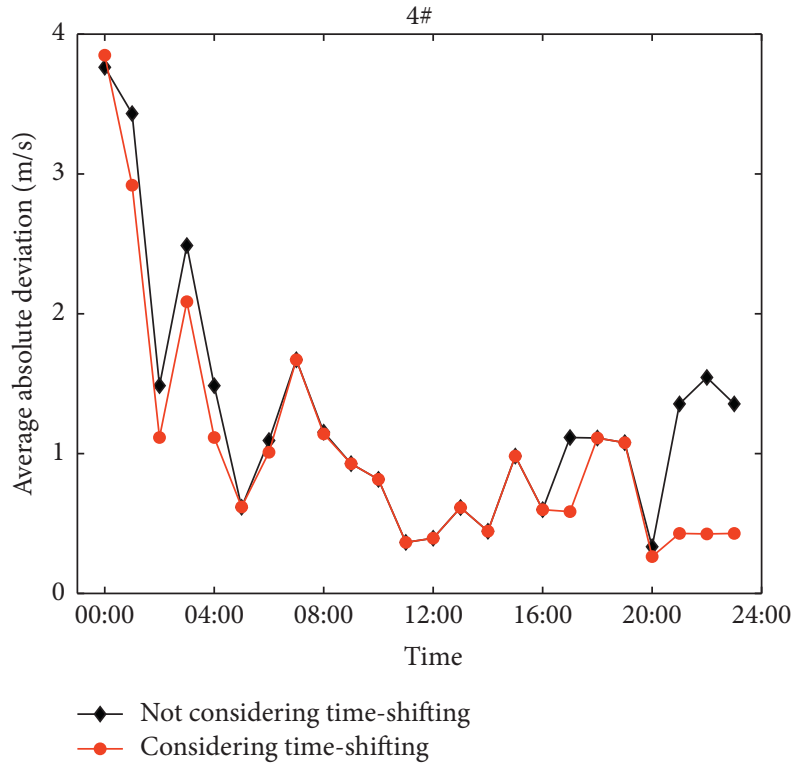

(c)

FIgURe 16: Average absolute deviations of wind speed sequences under two conditions at each point.

sequences are more similar to the actual wind speed sequences in all time windows if the time-shifting is considered.

In order to study the influence of time-shifting on the wind power generation system, the measured wind speed sequence and the calculated wind speed sequences under two conditions (considering time-shifting or not) at each point are converted to the power sequences based on the theoretical power curve of the wind turbine, respectively; then the total output of wind turbines at points 1 to 4 is obtained by adding the output of wind turbine at each point. The total power sequences of four wind turbines calculated by the measured wind speed sequence and the wind speed sequences of whether considering time-shifting are shown in Figure 17. It can be seen that the calculated total power is more similar to the actual power when the time-shifting is considered. To further illustrate the necessity of timeshifting, the influence of time-shifting is quantified from two perspectives: one is the volatility of the total power sequence and the other is the generation of four wind turbines. Fluctuation ratio (FR) [29] is used to evaluate the volatility of the power sequence in this paper. The calculation method of FR is shown in the following equation:

$$
\mathrm{FR}=\frac{\sum_{t=1}^{N-1}\left|(P(t+1)-P(t)) / P_{c a p}\right|}{N-1},
$$

where $P(t)$ is the power of wind turbines at time $t$ and $P_{\text {cap }}$ is the rated power of wind turbines, $8.02 \mathrm{MW}$ in this study.

The total power generation and the volatility of the power sequence of four wind turbines are calculated through the wind speed sequences under two conditions, and the results are compared with the results when using the measured wind speed sequences, as shown in Table 5. As can be seen from the table, if the time-shifting is taken

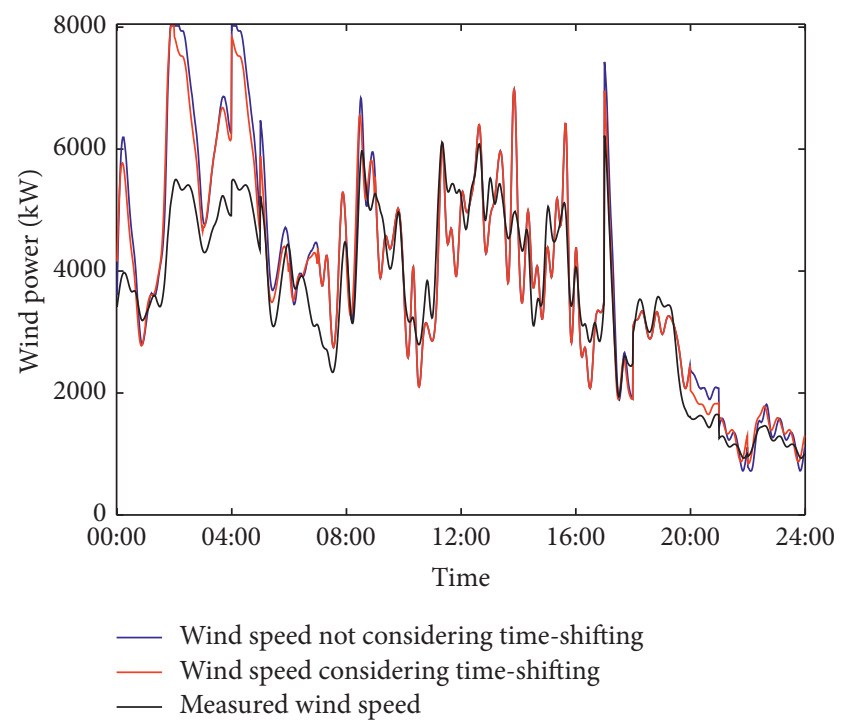

FIGURE 17: The power sequences of four wind turbines of whether considering time-shifting.

into account, the calculated daily power generation is closer to the actual generation, and the volatility of the calculated power sequence is more similar to that of the actual power sequence. The aggregation characteristics of wind turbines output are reflected. Compared with the actual daily power generation, the deviation of the calculated generation is $8.6 \%$ if the time-shifting is considered and is $10.6 \%$ if the time-shifting is not considered. Compared with the volatility of the actual power sequence, the deviation of the volatility of the calculated power sequence is $70.2 \%$ if the time-shifting is considered and is $77.2 \%$ if the time-shifting is not considered. 
TABLE 5: Comparison of wind power output under two conditions.

\begin{tabular}{|c|c|c|c|c|c|}
\hline \multicolumn{3}{|c|}{ Power generation $(\mathrm{MW} \cdot \mathrm{h})$} & \multicolumn{3}{|c|}{ Power volatility } \\
\hline $\begin{array}{l}\text { Not considering time- } \\
\text { shifting }\end{array}$ & $\begin{array}{l}\text { Considering time- } \\
\text { shifting }\end{array}$ & Measured & $\begin{array}{c}\text { Not considering time- } \\
\text { shifting }\end{array}$ & $\begin{array}{l}\text { Considering time- } \\
\text { shifting }\end{array}$ & Measured \\
\hline 96.51 & 94.77 & 87.28 & 0.0101 & 0.0097 & 0.0057 \\
\hline
\end{tabular}

\section{Conclusions}

A quantification method of wind speed time-shifting at different spatial positions based on wind process is proposed in this paper. As a result of this study, the following conclusions can be drawn:

(1) The proposed evaluation method can effectively achieve the quantitative analysis of the time-shifting characteristics of wind speed sequences at different spatial positions. Furthermore, the results of the proposed method can be continuously improved according to the actual wind conditions and could be applied to any spatial scale.

(2) The time-shifting effect between wind speed sequences increases with the distance when points are located in the flat terrain. But when points are located in the hilly terrain, the time-shifting effect does not show a more obvious trend with the increase of distance.

(3) The median delay time of wind speed sequences increases and the frequency distribution of the delay time becomes more dispersed with the increase of distance, no matter what terrain the points are located in.

(4) The wind speed at the downstream location could be larger than that at the upstream location in the wind farm when points are located in the hilly terrain.

(5) $x$-Means clustering method can achieve the effective division of wind processes, and the time-shifting characteristics of different wind processes are quite various.

(6) The calculated output of wind turbines is more similar to the actual output in the wind power system if the time-shifting characteristics are taken into account.

The time-shifting characteristics of wind speeds are studied when the wind direction is consistent with the relative position of points in this paper. In future research, the proposed evaluation method will be applied to analyze the time-shifting characteristics of wind speeds in full wind directions to better grasp the spatiotemporal coupling relationship of wind speeds at multiple scales.

\section{Data Availability}

The data used to support the findings of this paper are available upon request from the corresponding author.

\section{Conflicts of Interest}

The authors declare that there are no conflicts of interest regarding the publication of this paper.

\section{Acknowledgments}

This work was supported by the National Key Research and Development Program of China (2017YFE0109000) and the National Natural Science Foundation of China (U1765201 and 51707063).

\section{References}

[1] Y. Liu, Y. Ye, X. Chen, H. Li, and Y. Huang, "Robust dayahead dispatch for integrated power-heat-gas microgrid considering wind power uncertainty," Mathematical Problems in Engineering, vol. 2020, no. 9, Article ID 4215906, 12 pages, 2020.

[2] Y. Zhu, X. Liu, Y. Zhai, and R. Deng, "Monthly unit commitment model and algorithm with renewable energy generation considering system reliability," Mathematical Problems in Engineering, vol. 2019, Article ID 3835296, 9 pages, 2019.

[3] C. Lucheroni, J. Boland, and C. Ragno, "Scenario generation and probabilistic forecasting analysis of spatio-temporal wind speed series with multivariate autoregressive volatility models," Applied Energy, vol. 239, pp. 1226-1241, 2019.

[4] M. Cellura, G. Cirrincione, A. Marvuglia, and A. Miraoui, "Wind speed spatial estimation for energy planning in sicily: a neural kriging application," Renewable Energy, vol. 33, no. 6, pp. 1251-1266, 2008.

[5] S. Z. Moghaddam, "Generation and transmission expansion planning with high penetration of wind farms considering spatial distribution of wind speed," Electrical Power and Energy Systems, vol. 106, pp. 232-241, 2019.

[6] C. M. Grams, R. Beerli, S. Pfenninger, I. Staffell, and H. Wernli, "Balancing Europe's wind-power output through spatial deployment informed by weather regimes," Nature Climate Change, vol. 7, no. 8, pp. 557-562, 2017.

[7] W. Zheng, Research on Wind Speed Forecasting of Regional Wind Farm Group Based on Spatio-Temporal Correlation, North China Electric Power University, Beijing, China, 2014.

[8] S. She, Z. Li, and X. Cai, "Research on wind speed distribution model of wind farm based on its dynamic space-time relation," Power System Technology, vol. 38, no. 6, pp. 1432-1438, 2014.

[9] C. Zeng, L. Ye, and Y. Zhao, "Spatial model for short term wind power prediction considering wake effects," Power System Protection and Control, vol. 40, no. 24, pp. 59-142, 2012.

[10] L. Ye, Y. Zhao, C. Zeng, and C. Zhang, "Short-term wind power prediction based on spatial model," Renewable Energy, vol. 101, pp. 1067-1074, 2017.

[11] Y. L. Pichugina, R. M. Banta, T. Bonin et al., "Spatial variability of winds and HRRR-NCEP model error statistics at three Doppler-lidar sites in the wind-energy generation region of the Columbia river basin," Journal of Applied Meteorology and Climatology, vol. 58, no. 8, pp. 1633-1656, 2019. 
[12] F. Liu, F. Sun, W. Liu et al., "On wind speed pattern and energy potential in China," Applied Energy, vol. 236, pp. 867-876, 2019.

[13] J. Li and X. Yu, "Onshore and offshore wind energy potential assessment near Lake Erie shoreline: a spatial and temporal analysis," Energy, vol. 147, pp. 1092-1107, 2018.

[14] J. Bosch, I. Staffell, and A. D. Hawkes, "Temporally explicit and spatially resolved global offshore wind energy potentials," Energy, vol. 163, pp. 766-781, 2018.

[15] Y. Chen, S. Zhang, W. Zhang, J. Peng, and Y. Cai, "Multifactor spatio-temporal correlation model based on a combination of convolutional neural network and long short-term memory neural network for wind speed forecasting," Energy Conversion and Management, vol. 185, pp. 783-799, 2019.

[16] K. Philippopoulos and D. Deligiorgi, "Application of artificial neural networks for the spatial estimation of wind speed in a coastal region with complex topography," Renewable Energy, vol. 38, no. 1, pp. 75-82, 2012.

[17] M. S. Miranda and R. W. Dunn, "Spatially correlated wind speed modelling for generation adequacy studies in the UK," in Proceedings of the 2007 IEEE Power Engineering Society General Meeting, Tampa, FL, USA, June 2007.

[18] J. Park and D.-H. Jang, "Application of MK-PRISM for interpolation of wind speed and comparison with co-kriging in South Korea," GIScience \& Remote Sensing, vol. 53, no. 4, pp. 421-443, 2016.

[19] G. Ren, J. Wan, J. Liu, and D. Yu, "Assessing temporal variability of wind resources in China and the spatial correlation of wind power in the selected regions," Journal of Renewable and Sustainable Energy, vol. 12, Article ID 13302, 2020.

[20] Z. Q. Xie, T. Y. Ji, M. S. Li, and Q. H. Wu, "Quasi-Monte Carlo based probabilistic optimal power flow considering the correlation of wind speeds using copula function," IEEE Transactions on Power Systems, vol. 33, no. 2, pp. 2239-2247, 2018.

[21] X. Hui, Calculation of Available Transfer Capability Considering Temporal and Spatial Correlation in Wind Power Integrated System, Northeast Electric Power University, Jilin, China, 2018.

[22] G. Ren, J. Wan, J. Liu, and D. Yu, "Spatial and temporal assessments of complementarity for renewable energy resources in China," Energy, vol. 177, pp. 262-275, 2019.

[23] M. Zeng, J.-h. Li, Q.-h. Meng, and X.-n. Zhang, “Temporalspatial cross-correlation analysis of non-stationary nearsurface wind speed time series," Journal of Central South University, vol. 24, no. 3, pp. 692-698, 2017.

[24] S. T. Frandsen, H. E. Jørgensen, R. Barthelmie et al., "The making of a second-generation wind farm efficiency model complex," Wind Energy, vol. 12, no. 5, pp. 445-458, 2009.

[25] M. B. Imamoglu, M. Ulutas, and G. Ulutas, "A new reversible database watermarking approach with firefly optimization algorithm," Mathematical Problems in Engineering, vol. 2017, Article ID 1387375, 14 pages, 2017.

[26] Y. Kang, Z. Zhang, and X. Guo, "Portfolio risk analysis in electricity market based on copula approach," Power System Protection and Control, vol. 40, no. 6, pp. 50-56, 2012.

[27] Z. Jiang and J. He, "Method of fusion diagnosis for dam service status based on joint distribution function of multiple points," Mathematical Problems in Engineering, vol. 2016, Article ID 9049260, 10 pages, 2016.

[28] D. Pelleg and A. Moore, " $X$-means: extending $K$-means with efficient estimation of the number of clusters," in Proceedings of the Seventeenth International Conference on Machine Learning, Stanford, CA, USA, July 2000.

[29] S. Han, L.-n. Zhang, Y.-q. Liu et al., "Quantitative evaluation method for the complementarity of wind-solar-hydro power and optimization of wind-solar ratio," Applied Energy, vol. 236, pp. 973-984, 2019. 